\title{
ON NUMERICAL DENSITY APPROXIMATIONS OF SOLUTIONS OF SDES WITH UNBOUNDED COEFFICIENTS
}

\author{
LINGHUA CHEN, ESPEN ROBSTAD JAKOBSEN, AND ARVID NAESS
}

\begin{abstract}
We study a numerical method to compute probability density functions of solutions of stochastic differential equations. The method is sometimes called the numerical path integration method and has been shown to be fast and accurate in application oriented fields. In this paper we provide a rigorous analysis of the method that covers systems of equations with unbounded coefficients. Working in a natural space for densities, $L^{1}$, we obtain stability, consistency, and new convergence results for the method, new well-posedness and semigroup generation results for the related Fokker-Planck-Kolmogorov equation, and a new and rigorous connection to the corresponding probability density functions for both the approximate and the exact problems. To prove the results we combine semigroup and PDE arguments in a new way that should be of independent interest.
\end{abstract}

\section{INTRODUCTION}

Over the past decades there has been a large number of publications in the field of stochastic dynamics and its various application areas - including physics, biology, engineering, and finance [34, 2, 11]. In this field, the response of dynamical systems to stochastic excitation is studied, and the typical model is a (system of) stochastic differential equations (SDEs) of the form

$$
\left\{\begin{array}{l}
d Y_{t}=b\left(t, Y_{t}\right) d t+\sigma\left(t, Y_{t}\right) d B_{t}, \\
Y_{0}=Z,
\end{array}\right.
$$

where $b: \mathbb{R}_{+} \times \mathbb{R}^{d} \rightarrow \mathbb{R}^{d}, \sigma: \mathbb{R}_{+} \times \mathbb{R}^{d} \rightarrow \mathbb{R}^{d \times n}, B_{t}$ is an $n$-dimensional Brownian motion, and the initial data $Z$ is a random variable in $\mathbb{R}^{d}$ independent of $B_{t}$. The solution $Y_{t}$ of the SDE is a state space process in $\mathbb{R}^{d}$.

In most cases the solutions of such problems must be computed numerically, and various discrete approximation methods are widely used in many application areas [24]. There are two main approaches: (i) Path-wise approximations of the SDE based on stochastic simulation, and (ii) approximations of the statistics or distributions of the SDE. The first approach is more efficient in high dimensions and the second in low dimensions. For path-wise approximations we refer to [24] for the

Date: July 9, 2018.

1991 Mathematics Subject Classification. 60H35, 65M12, $47 \mathrm{D} 07$.

Key words and phrases. Stochastic Differential Equations, Numerical Method, Path Integration, Density Tracking, Probability Density, Semigroup Generation, Convergence.

E. R. Jakobsen is partially supported by the NFR Toppforsk project 250070 , Waves and Nonlinear Phenomena (WaNP). The authors are thankful to Harald Hanche-Olsen and Peter Lindqvist for their valuable inputs to Proposition 3.4 and Lemma 5.6, respectively. 
classical literature and e.g. to [31] and references therein for some promising recent developments. Approach (ii) is deterministic and based on approximating the forward or backward Kolmogorov partial differential equations. In this paper we study a method of the second type, called the numerical path integration method $[36,29,39,41,40,10]$ or density tracking method [5]. This method is an explicit deterministic iteration scheme that produces approximate probability density functions (PDFs) for the solution of the SDE. The iteration step is based on a short time approximation of the SDE. For simplicity we use here the strong Euler-Maruyama method, the most basic numerical method for SDEs. The convergence of our path integration method is therefore equivalent to the convergence of the PDFs of the Euler-Maruyama method.

The path integration/density tracking approach (i.e. simulating the PDFs) enjoys several favorable properties. First, it introduces an extra perspective to the system, which enables deeper insights and invites broader mathematical tools. Secondly, as an explicit method, one can formally implement the path integration algorithm on a vast number of scenarios. Since the formulation is deterministic, it is also free from perturbation by extreme outcomes during stochastic simulation. Finally, the result of the method is an explicit density function rather than bundle of random paths. This means that many characteristics of the system become more transparent, and can be captured and displayed by e.g. visualisation methods.

The numerical path integration method has been applied in many fields, including financial mathematics $[29,39,40,10]$. Many of these studies show that it can provide highly accurate numerical solutions $[36,41,35,5]$. Even though convergence problems have been reported in some cases, cf. Section 7.1 in [35], little or no emphasis has been devoted to conditions for convergence of the method in the path integration literature. A very natural and relevant mode of convergence for this method is strong $L^{1}$-convergence of the resulting densities. Such convergence seems not to be a direct consequence of either strong or weak convergence of the EulerMaruyama scheme. In fact, $L^{1}$-convergence of densities implies weak convergence of processes but the converse is not true in general. Nevertheless, very recently there are some results for one-dimensional problems in [5], and when the coefficients are bounded, $L^{1}$-convergence results follow from some of very precise error expansions for densities of the Euler-Maruyama method, e.g. in [4, 20, 19], or from so-called Feynman formulas in mathematical physics [8]. More on this below. However from an application point of view, it is important to consider also models with unbounded coefficients, since they naturally appear in many of the papers and applications mentioned above. ${ }^{1}$

The aim of this paper is to provide a rigorous analysis of method in the path integration setting along with the $L^{1}$-convergence of the PDFs it produces in the case of unbounded coefficients. Specifically, we obtain stability, consistency, and new convergence results for the method, new well-posedness and semigroup generation results for the associated Fokker-Planck-Kolmogorov (FPK) equation, and a new and rigorous connection to the corresponding probability density functions for both

\footnotetext{
${ }^{1}$ By a change of variables, it is often possible to reduce to a problem with bounded coefficients and then use existing $L^{1}$ convergence results. However going back to the original variables, we would then get a weaker form of convergence, one that no longer implies $L^{1}$ convergence.
} 
the approximate and exact problems. In particular, our $L^{1}$ semigroup generation result for our rather general elliptic FKP operator seems to be new.

We also investigate in details the method applied to the Ornstein-Uhlenbeck process which has unbounded drift. Using explicit transition densities and direct computations, we show that the method (i) converge in the general case and (ii) converge with a linear rate if additional moment conditions are assumed. The second result is consistent with results for bounded coefficients in e.g. [4, 20, 19]. Even though we do not have a counter example, the very explicit proof seems to indicate that convergence rates can only be obtained under some additional moment assumptions on the densities.

We study the numerical path integration method from a PDE and semigroup point of view, an approach which seems to be new to this setting at least when it comes to the convergence results. The main idea is to show that (i) the FPK operator corresponding to the SDE generates a semigroup, (ii) the iteration of the discrete path integration operator does converge to this semigroup (the solution of the FPK equation), and finally (iii) that convergence of the densities follows from these results. For (i), we prove that the FKP operator and its adjoint are dissipative and then use the Lumer-Phillips theorem to prove that the FPK operator generates a strongly continuous contraction semigroup on $L^{1}\left(\mathbb{R}^{d}\right)$. To prove the convergence results in (ii), we use the Chernoff product formula which is a generalisation of the well-known Kato-Trotter product formula. Note that our approach to (ii) is similar to the approach for Feynman formulas in [8].

A main difficulty is then to prove the semigroup generation in our setting of unbounded coefficients. Classical results usually assume the coefficients to be bounded, cf. the discussion in Chapter 11 of [28]. Further complications comes from the fact that we have to work in the space $L^{1}$ which is not reflexive and the adjoint space $L^{\infty}$ which is not separable. In fact one of the most difficult parts of our paper is to show that the adjoint operator is dissipative on $L^{\infty}$. To do this we develop a new and non-trivial argument using ideas from viscosity solution theory [12], the weak Bony maximum principle [6,30], and recent elliptic regularity results from [42]. This argument could be of independent interest.

Other authors have obtained semigroup generation results for similar problems with different techniques. Yet, most work seems to be devoted to the generation problems for the adjoint of the FPK operator in various situations. For instance [13] is concerned with the space of bounded (continuous) functions and unbounded coefficients, while in [1] the authors give results in $L^{1}$ for bounded coefficients. The author in [9] considered degenerate operators where the coefficients have bounded first and second derivatives. The result closest to our case seems to be found in [15] where the drift term had to be dominated by the "square root" of the diffusion term and no zero order term is allowed. A semigroup generation result of the adjoint operators on $L^{1}$ is proved there. However, we cannot use the results in [15] in this paper since our non-divergence form FPK operators are equivalent to divergence form operators with additional unbounded zero order terms.

In addition to providing rigorous results for the numerical path integration method, this paper seems to represent the first attempt to use semigroup and PDE arguments to obtain strong convergence results for probability densities of SDE approximations 
(but see also [8]). It is an interesting question how far such methods can be pushed compared to other methods. As far as we know, two other methods are described in the literature: A parametrix approach of Konakov et al. [25, 26, 33], and an approach based on Malliavin calculus developed by Talay, Bally, et al., see e.g. [4, $20,3]$ and references therein. In the parametrix approach, a weighted $L^{\infty}$-estimate on the convergence of (transition probability) densities, as well as non-asymptotic bounds for the densities [33], is obtained under uniform ellipticity assumption and boundedness of coefficients. In the Malliavin approach various error expansions and estimates are obtained under boundedness, certain smoothness, and ellipticity or Hörmander conditions.

Under additional assumptions, the $L^{1}$-convergence of densities of the Euler-Maruyama scheme can be obtained from some of these Malliavin results. In [4] a linear convergence rate was obtained for SDEs with smooth and bounded coefficients ${ }^{2}$, but the behavior of the estimate is unclear for small time. In [20] the estimate was improved but it would still blow up as $t \rightarrow 0$. Later the authors of [19] derived very accurate estimates that would lead to a linear rate which is uniform for small time. At the same time they lowered the regularity requirements of the coefficients to $C_{b}^{1,3}$. For the Euler-Maruyama method, the estimates of $[4,20,19]$ requires bounded coefficients and uniform ellipticity. According to Remark 2.22 of [3], the ellipticity requirement can be relaxed to a so-called weak Hörmander condition when coefficients belong to $C_{b}^{\infty}$. In this case, the results of [3] give $L^{1}$-convergence for fixed times (but no estimates) of densities/PDFs for the Euler-Maruyama scheme.

To summarise, in our approach to study properties and convergence of the numerical path integration method we obtain the following results: New semigroup generation and well-posedness results for the FPK equation, allowing for coefficients with linear growth and $C^{4}$ differentiability, no invariant measure is required, the growth of drift and diffusion terms are not necessarily related to each another, and the initial data is not required to have any finite moments or differentiability. Under the same conditions, we prove $L^{1}$ convergence, locally uniformly in time (so no deterioration for small time). To our knowledge, this seems to be the first general strong $L^{1}$ convergence result for densities/PDFs of SDEs with unbounded coefficients. Even though we do not obtain any rate of convergence or error expansion in general, by studying the Ornstein-Uhlenbeck process, we find indications that no error estimate may exist without additional (moment) assumptions on the densities/PDFs. Hence when the coefficients are unbounded, the best result for general densities could be mere convergence without any rate.

We also emphasise that our results connects the mild solution of the FPK equation and the PDF of the SDE, and that existence and convergence of the latter follows from the existence and convergence (by semigroup methods) of the former. More refined existence results for PDFs have been obtained by probabilistic methods, we refer to e.g. $[7,16,21,22]$ and references therein.

Layout and Notation. The rest of this paper is laid out as follows. In Section 2 we state our assumptions and the main results. Included is a discussion of the connection between SDEs, FPK equations and densities and the definition of the

\footnotetext{
${ }^{2}$ The coefficients are bounded in [4]. This follows from their condition (H), estimate (6), and footnote 1. In particular, in [4] the number $\gamma_{0}$, introduced by (1.10) in [27], is zero.
} 
path integration method. At the end of the section, we discuss our results and give some examples. The rest of the paper is then devoted to the proofs of these results. In Section 3 we prove the well-posedness of the FPK equation, the connection to probability densities, and the convergence result - assuming the semi-group generation and a strong $L^{1}$-consistency result for the path integration method, which are proved in Section 4 and Section 5, respectively. At the end, Section B is an appendix to the calculation of the convergence rate of Example 2.13.

The following notation and abbreviations are used $\|\cdot\|_{1}:=\|\cdot\|_{L^{1}\left(\mathbb{R}^{d}\right)},\|\cdot\|_{\infty}:=$ $\|\cdot\|_{L^{\infty}\left(\mathbb{R}^{d}\right)}, \partial_{t}:=\frac{\partial}{\partial t}, \nabla:=\left(\frac{\partial}{\partial x_{1}}, \cdots, \frac{\partial}{\partial x_{d}}\right)^{T}=:\left(\partial_{1}, \cdots, \partial_{d}\right)^{T}$, ess inf is the essential infimum, $\mathbb{E}$ denotes the expectation; $C_{b}^{k}, C_{c}^{\infty}, \mathcal{D}^{\prime}$ the spaces of functions with bounded continuous derivatives up to $k$-th order, smooth compactly supported functions, and distributions $\left(\mathcal{D}^{\prime}\right.$ is the dual of $\left.C_{c}^{\infty}\right)$ respectively, $C\left([0, T] ; L^{1}\left(\mathbb{R}^{d}\right)\right)$ the space of functions $u(x, t)$ such that $\sup _{t \in[0, T]}\|u(\cdot, t)\|_{1}<\infty$, and for all $t \in$ $[0, T],\|u(\cdot, s)-u(\cdot, t)\|_{1} \rightarrow 0$ as $s \rightarrow t$. PDF - probability density function, SDE stochastic differential equation, FPK - Fokker-Planck-Kolmogorov.

\section{Main Results}

In this section we formulate the discrete path integration method, state the assumptions and main results, and provide a discussion and examples. We restrict ourselves to time-homogeneous SDEs, i.e. equations of the form

$$
\left\{\begin{array}{l}
d Y_{t}=b\left(Y_{t}\right) d t+\sigma\left(Y_{t}\right) d B_{t}, \\
Y_{0}=Z,
\end{array}\right.
$$

where $b=\left(b_{1}, \cdots, b_{d}\right)^{T}: \mathbb{R}^{d} \rightarrow \mathbb{R}^{d}$ and $\sigma=\left(\sigma_{i j}\right)_{d \times n}: \mathbb{R}^{d} \rightarrow \mathbb{R}^{d \times n}$ are functions, $B_{t}$ a $d$-dimensional Brownian motion, and $Z$ is a random variable in $\mathbb{R}^{d}$.

Under suitable assumptions (cf. [37] or [38]), the solution $Y_{t}$ of (2.1), is a Markov process, with infinitesimal generator $A^{*}$ defined as

$$
A^{*} \phi:=\sum_{i=1}^{d} b_{i}(x) \partial_{i} \phi(x)+\frac{1}{2} \sum_{i, j=1}^{d} a_{i j}(x) \partial_{i} \partial_{j} \phi(x) \quad \text { for all } \quad \phi \in C_{c}^{\infty}\left(\mathbb{R}^{d}\right),
$$

where

$$
a:=\sigma \sigma^{T}=:\left(a_{i j}\right)_{d \times d} .
$$

Moreover, $Y_{t}$ has a PDF $u(t, x)$ - a non-negative $L^{1}$ function $u$ such that

$$
\mathbb{E} \phi\left(Y_{t}\right)=\int_{\mathbb{R}^{d}} \phi(x) u(t, x) d x \quad \text { for all } \phi \in C_{b}^{0}\left(\mathbb{R}^{d}\right) .
$$

Via an adjoint argument, $u$ formally satisfies the FPK equation

$$
\begin{cases}\partial_{t} u(t, x)=A u(t, x), & x \in \mathbb{R}^{d}, t>0, \\ u(0, x)=u_{0}(x), & x \in \mathbb{R}^{d},\end{cases}
$$

where $u_{0}$ is the PDF of $Z$ and $A$ the adjoint of $A^{*}$,

$$
A v(x):=-\sum_{i=1}^{d} \partial_{i}\left(b_{i}(x) v(x)\right)+\frac{1}{2} \sum_{i, j=1}^{d} \partial_{i} \partial_{j}\left(a_{i j}(x) v(x)\right) .
$$


The evolution of the $u$ can be regarded as the action of the propagation operator (or semigroup) $P_{s}$ defined by

$$
u(y, t+s)=\left[P_{s} u(\cdot, t)\right](y) \quad \text { for all } \quad t, s>0, y \in \mathbb{R}^{d} .
$$

Let us state our assumptions.

(C1) $b: \mathbb{R}^{d} \rightarrow \mathbb{R}^{d}$ and $\sigma: \mathbb{R}^{d} \rightarrow \mathbb{R}^{d \times n}$ are $C^{4}$ functions, and there exists a constant $K>0$ such that for all $x \in \mathbb{R}^{d}, j=1, \ldots, n$, and $i, k=1, \ldots, d$,

$$
\left|\partial_{k} \sigma_{i j}(x)\right|+\left|\partial_{k} b_{i}(x)\right| \leq K \text {. }
$$

(C2) There exists $\alpha>0$ such that for all $x, y \in \mathbb{R}^{d}$,

$$
y^{T} a(x) y \geq \alpha|y|^{2} .
$$

(C3) $Z$ and $B_{t}$ are independent, and $Z$ has a PDF $u_{0}$, i.e. $u_{0} \geq 0$ and $\left\|u_{0}\right\|_{1}=1$.

Remark 2.1. Standard strong well-posedness of the SDE (see below) requires only Lipschitz coefficients. The additional regularity and the uniform ellipticity condition (C2) is needed to study the PDFs of the SDEs and path integration method (cf. proofs of Proposition 2.10 and Lemma 5.8). Similar but stronger assumptions, including bounded coefficients, are used by all other papers discussing densities of SDE approximations, see the introduction and e.g. [19].

The following result is then classical, cf. Theorem V.7 in [38].

Proposition 2.2. Assume (C1) and (C3). Then the SDE (2.1) has a unique strong solution $Y_{t}$.

Now we give our first main result - well-posedness for the FPK equation (2.4).

Theorem 2.3. Assume (C1), (C2), and $u_{0} \in L^{1}\left(\mathbb{R}^{d}\right)$. Then the FPK equation (2.4) has a unique (mild) solution $u \in C\left([0, T] ; L^{1}\left(\mathbb{R}^{d}\right)\right)$.

The derivation of FPK equation (2.4) was formal. Yet our second main result confirms that the probability distribution of (2.1) coincides with the (mild) solution of FPK equation (2.4).

Theorem 2.4. Assume (C1) - (C3), and let $Y_{t}$ and $u(t, x)$ be the solutions of (2.1) and (2.4) respectively. Then (2.3) holds and $u(t, \cdot)$ is the PDF of $Y_{t}$.

The proofs of Theorems 2.3 and 2.4 will be given in the next section.

Remark 2.5. (a) The proof shows that Theorem 2.3 still holds if the $C^{4}$ regularity in assumption (C1) is reduced to $b \in C^{1}$ and $\sigma \in C^{2}$.

(b) A difference between Theorem 2.3 and existing results is that we allow (and need to allow) the zero order coefficient to be unbounded, cf. e.g. Section 4 in [15].

(c) The solution $u$ we obtain in Theorem 2.3 is a mild solution of (2.4) (cf. [14]). In fact we have more regularity than stated since $u(\cdot, t)$ belongs to the domain of $A$. A limit argument immediately shows that $u$ is also a distributional solution of (2.4). In view of uniform ellipticity of $A$ and smoothness of its coefficients, it is standard to prove higher regularity and that $u$ is a classical solution. 
We now introduce and analyse the discrete path integration method. It is a method for computing approximations of the PDF of solution process of (2.1) by:

(i) Finding a short-time approximation $\bar{P}_{\tau}$ of the propagator/semigroup $P_{\tau}$, and

(ii) computing the approximation $\bar{u}_{n}$ at time $t=n \tau$ using the explicit iteration

$$
\bar{u}_{n+1}(x)=\left(\bar{P}_{\tau} \bar{u}_{n}\right)(x) \text { for } n=0,1, \ldots
$$

The most obvious way to find a $\bar{P}_{\tau}$ is through a time-discretization of the underlying SDE. For simplicity, we consider here the Euler-Maruyama method [24], the most basic and widely used SDE approximation scheme: Fix a time step $\tau>0$ and let $\Delta B_{n}:=B_{n \tau}-B_{(n-1) \tau}$, and define the Markov chain approximation $X_{n}$ of the solution process $Y_{t}$ of the $\operatorname{SDE}(2.1)$ by

$$
\left\{\begin{array}{l}
X_{0}=Z, \\
X_{n+1}=X_{n}+b\left(X_{n}\right) \tau+\sigma\left(X_{n}\right) \Delta B_{n}, \quad n=0,1, \ldots
\end{array}\right.
$$

In the rest of the paper we will use the following notation.

Definition 2.6. (i) $\quad \bar{u}_{n}$ is the PDF of the solution $X_{n}$ of (2.8).

(ii) $\quad \bar{P}_{\tau}$ is the one step propagator of $\bar{u}_{n}$, i.e.

$$
\bar{u}_{n+1}(y)=\left(\bar{P}_{\tau} \bar{u}_{n}\right)(y) \quad \text { for all } \quad y \in \mathbb{R}^{d}, \tau>0, n \geq 0 .
$$

(iii) $\quad \bar{k}(y, x, \tau)$ is the transition kernel of $\bar{P}_{\tau}$ - a non-negative $L^{1}$ function satisfying

$$
\left(\bar{P}_{\tau} \bar{u}_{n}\right)(y)=\int_{\mathbb{R}^{d}} \bar{k}(y, x, \tau) \bar{u}_{n}(x) d x \quad \text { for all } \quad y \in \mathbb{R}^{d}, \tau>0, n \geq 0 .
$$

Since $\Delta B_{n}$ is a multivariate Gaussian variable, we have the following classical result.

Lemma 2.7. Assume $a:=\sigma \sigma^{T}$ is strictly positive definite, and let $\bar{k}$ be given by Definition 2.6. Then for all $x, y \in \mathbb{R}^{d}$ and $\tau>0$,

$$
\mathcal{F}_{y} \bar{k}(\xi, x, \tau)=\exp \left\{i \xi^{T}(x+b(x) \tau)-\frac{1}{2} \xi^{T} a(x) \xi\right\},
$$

where $\mathcal{F}$ denote the Fourier transform, and

$$
\bar{k}(y, x, \tau)=\frac{\exp \left\{-\frac{1}{2}(y-(x+b(x) \tau))^{T}(\tau a)^{-1}(x)(y-(x+b(x) \tau))\right\}}{(2 \pi)^{2 / d}|\operatorname{det}(\tau a(x))|^{1 / 2}} .
$$

Existence and uniqueness of the solution $X_{n}$ come for free, since the iteration (2.8) is explicit. In view of Lemma 2.7, we then have the following result.

Proposition 2.8 (Well-posedness). Assume (C1) - (C3). Then there exists a unique PDF $\bar{u}_{n}$ of the solution $X_{n}$ of (2.8). Moreover, $\bar{u}_{n}$ is explicitly given by (2.7), (2.9), and (2.10).

Note that $(\mathrm{C} 2)$ implies that $a(x)$ is strictly positive definite. A classical computation using (2.10) then shows that $\|\bar{k}(\cdot, x, \tau)\|_{1}=1$, and hence (see the next Proposition) that $\left\|\bar{P}_{\tau}\right\|=1$. Proofs can be found e.g. in the discussion of Markov operators in [28]. 
Proposition 2.9 ( $L^{1}$-stability). Assume (C2), $\tau>0, u \in L^{1}\left(\mathbb{R}^{d}\right)$, and $\bar{P}_{\tau}$ is given by Definition 2.6. Then $\left\|\bar{P}_{\tau} u\right\|_{1} \leq\|u\|_{1}$.

By linearity, we immediately get continuous dependence on the initial data,

$$
\left\|\bar{P}_{\tau}(u-v)\right\|_{1} \leq\|u-v\|_{1},
$$

and by iteration that the discrete path integration method is $L^{1}$-stable:

$$
\left\|\bar{u}_{n}\right\|_{1} \leq\left\|u_{0}\right\|_{1} \quad \text { for all } \quad n=1,2, \ldots
$$

Next we study the $L^{1}$-consistency of the discrete scheme.

Proposition 2.10 ( $L^{1}$-consistency). Assume (C1), (C2), $\tau>0$, and $u \in C_{c}^{\infty}\left(\mathbb{R}^{d}\right)$. Then there exists a constant $C>0$ such that

$$
\left\|\frac{\bar{P}_{\tau} u-u}{\tau}-A u\right\|_{1} \leq C \tau
$$

We prove this result in Section 4. Higher regularity of $b$ or $\sigma$ will not improve the (linear) rate in Proposition 2.10. It is the maximal rate for the problem, cf. Proposition 2.14 and its proof.

Finally we state the third main result of this paper, the $L^{1}$-convergence of the discrete path integration method.

Theorem 2.11 ( $L^{1}$-convergence). Assume (C1), (C2), $u_{0} \in L^{1}\left(\mathbb{R}^{d}\right), u(t, x)$ is the solution of FPK equation (2.4), and define $\bar{u}_{n}(t, x):=\left(\bar{P}_{t / n}^{n} u_{0}\right)(x)$. Then

$$
\lim _{n \rightarrow \infty} \sup _{t \in[0, T]}\left\|u(\cdot, t)-\bar{u}_{n}(\cdot, t)\right\|_{1}=0 .
$$

Remark 2.12. (a) Since the PDFs in general are (non-negative) $L^{1}$-functions, strong $L^{1}$-convergence is a very natural mode of convergence to consider.

(b) By assumption the initial PDF $u_{0}$ is only required to belong to $L^{1}$. It is not required to have any finite moments or differentiability to obtain an $L^{1}$-convergence, and the convergence is uniform in time on $[0, T]$ for any $T>0$.

(c) $L^{1}$-convergence of PDFs is not a direct consequence of either strong or weak convergence of the solution process. It is strictly stronger than weak convergence of the corresponding process by Proposition A.1 and Example A.2 in Appendix A.

When the coefficients are also bounded, many authors have obtained not only convergence but even error estimates and error expansions for densities of the EulerMaruyama scheme (see the introduction).

In the unbounded coefficients case however, there may not be any general $L^{1}$ error bound without extra moment assumptions on the density, and hence mere convergence would be the best one can hope for. A first indication of this appears already in the Gaussian bounds on the derivatives of the SDE's transition probability density $p(t, x, y)$ in [27]. When the coefficients are unbounded, these bounds are no longer bounded in $x$, and hence can no longer be used to derive the same estimates of approximations of $p(t, x, y)$ as in the bounded case [4, 19]. Another indication is given by Example 2.13 below. In this example we study the Ornstein-Uhlenbeck process. We find both the exact and approximate transition kernels and compute 
explicitly the conditions for having either mere convergence in $L^{1}$ or a linear rate for the corresponding PDFs. In the latter case, we find that additional moment assumptions are needed (cf. Proposition 2.14).

Example 2.13. The Ornstein-Uhlenbeck process $Y_{t}$ is given by

$$
d Y_{t}=b Y_{t} d t+\sigma d B_{t} \quad \text { and } \quad Y_{0}=x,
$$

where $b, \sigma \in \mathbb{R}$ and $Y_{t}, B_{t}$ are processes in $\mathbb{R}$. The unique strong solution is

$$
Y_{t}=e^{b t}\left(x+\sigma \int_{0}^{t} e^{-b s} d B_{s}\right) .
$$

Due to the non-stochastic integrand in the Itô-integral it is easy to see that this process has a Gaussian law (cf. e.g. Section 11.5 in [14]),

$$
N\left(x e^{b t}, \frac{\sigma^{2}}{2 b}\left(e^{2 b t}-1\right)\right) \text {, }
$$

and hence the transition kernel is given by

$$
k(y, x, t)=\frac{1}{\sqrt{2 \pi \frac{\sigma^{2}}{2 b}\left(e^{2 b t}-1\right)}} \exp \left\{-\frac{\left(y-x e^{b t}\right)^{2}}{2 \frac{\sigma^{2}}{2 b}\left(e^{2 b t}-1\right)}\right\} .
$$

The corresponding Euler-Maruyama scheme with $\tau=t / n$ is

$$
\left\{\begin{array}{l}
X_{0}=x, \\
X_{n+1}=X_{n}+b X_{n} \tau+\sigma \Delta B_{n},
\end{array}\right.
$$

and has the following one step transition kernel by Lemma 2.7,

$$
\bar{k}(y, x, \tau)=\frac{1}{\sqrt{2 \pi \tau \sigma^{2}}} \exp \left\{-\frac{(y-(1+b \tau) x)^{2}}{2 \tau \sigma^{2}}\right\} .
$$

For $t=\tau$ small, it is clear that $\bar{k}(y, x, \tau)$ is close to $k(y, x, t)$.

For the convergence, we have the following result.

Proposition 2.14. Assume $b, \sigma \in \mathbb{R}, T>0$, and $u_{0} \in L^{1}(\mathbb{R})$. Then

$$
\lim _{n \rightarrow \infty} \sup _{t \in[0, T]}\left\|P_{t} u_{0}-\bar{P}_{t / n}^{n} u_{0}\right\|_{1}=0 .
$$

If in addition, either

$$
\int_{\mathbb{R}}\left|x u_{0}(x)\right| d x<\infty \quad \text { or } \quad \int_{\mathbb{R}}\left|x u_{0}^{\prime}(x)\right| d x<\infty,
$$

then there exist $C, N>0$, only depending on $\left(b, \sigma, T, u_{0}\right)$, such that

$$
\left\|P_{t} u_{0}-\bar{P}_{t / n}^{n} u_{0}\right\|_{1} \leq \frac{C t}{n} \quad \text { for all } n \geq N \text {. }
$$

The first part of Proposition 2.14 is consistent with Theorem 2.11, while the second part shows that a linear rate can be obtained under additional assumptions. The rate is consistent with estimates for SDEs with bounded coefficients, see e.g. Corollary 2.7 in [4] and Theorem 2.3 in [19].

We give a direct proof of Proposition 2.14 in Appendix B. Even though we do not have a counter example, the very explicit proof seems to indicate that convergence rates can only be obtained under additional assumptions on the densities. 
Remark 2.15. The Cauchy distribution $u_{0}(x)=\frac{1}{\pi\left(1+x^{2}\right)}$ has no moments, but still satisfies $\int_{\mathbb{R}}\left|x u_{0}^{\prime}(x)\right| d x<\infty^{3}$.

\section{Proofs of Theorems $2.3,2.4$, And 2.11}

We first prove Theorems 2.3 and 2.11 since the latter is needed in the proof of Theorem 2.4. We will use semi-group theory and we refer to [14] for more information and precise definitions of the concepts used below. A crucial step in the proof is to obtain the new generation result, Theorem 3.1. From the generation result we obtain a solution of the FPK equation (2.4), and Theorem 2.3 follows. Then we prove that FPK operator $A$ is dissipative. Armed with generation and dissipativity, we use the Chernoff product formula to show that the solution of the discrete path integration method converges to the solution of the FPK equation (2.4) and hence Theorem 2.11 follows. Finally we show Theorem 2.4, that the solution of (2.4) is just the PDF of the solution of (2.1).

In the rest of this section we let $A$ be defined in $(2.5)$ and take $D(A):=C_{c}^{\infty}\left(\mathbb{R}^{d}\right)$. First we state the generation result.

Theorem 3.1. Assume (C1) and (C2). Then $(A, D(A))$ is closable and its closure $(\bar{A}, D(\bar{A}))$ generates a contraction semigroup $P_{t}$ on $\left(L^{1}\left(\mathbb{R}^{d}\right),\|\cdot\|_{1}\right)$.

This result is relatively technical, and the proof is left to Section 5 .

Lemma 3.2. Assume (C1) and (C2). Then the semigroup $P_{t}$ from Theorem 3.1 is a strongly continuous semigroup on $\left(L^{1}\left(\mathbb{R}^{d}\right),\|\cdot\|_{1}\right)$.

Proof. The result follows by Proposition I.5.3 in [14] with $T_{t}=P_{t}$ if we can verify that there exist $\delta>0, M \geq 1$ and a dense subset $D \subset X$ such that

(1) $\left\|T_{t}\right\| \leq M$ for all $t \in[0, \delta]$,

(2) $\lim _{t \rightarrow 0+} T_{t} x=x$ for all $x \in D$.

Since $P_{t}$ is a contraction semigroup by Theorem 3.1, condition (1) is satisfied with $M=1$. Moreover, assumption (2) holds on the dense set $D=D(A)$, since by the definition of the generator $A$ :

$$
\lim _{t \rightarrow 0+}\left(P_{t} x-x\right)=\lim _{t \rightarrow 0+} t\left(P_{t} x-x\right) / t=\left(\lim _{t \rightarrow 0+} t\right) \lim _{t \rightarrow 0+} \frac{1}{t}\left(P_{t} x-x\right)=0 \cdot A x=0 .
$$

Hence (1) and (2) holds, and we conclude that $P_{t}$ is strongly continuous.

Proof of Theorem 2.3. In view of Theorem 3.1 and Lemma 3.2, the closure of $(A, D(A))$ generates a strongly continuous semigroup $P_{t}$ on $\left(L^{1}\left(\mathbb{R}^{d}\right),\|\cdot\|_{1}\right)$. Let $u(t, x):=\left(P_{t} u_{0}\right)(x)$, and note that $u \in C\left([0, T] ; L^{1}\left(\mathbb{R}^{d}\right)\right)$ by strong continuity. Then by e.g. Proposition II.6.4 in [14], $u$ is the unique mild solution of the FPK equation (2.4).

To proceed, we introduce the following definition.

\footnotetext{
${ }^{3}$ An absolutely continuous $L^{1}$-functions not satisfying either of the conditions: $u_{0}(x)=$ $\sum_{k \neq 0} \chi_{I_{k}}(x)\left(1-k^{2}|x-k|\right)$, where $I_{k}:=\left[k-1 / k^{2}, k+1 / k^{2}\right]$ and $k \in \mathbb{Z} \backslash\{0\}$.
} 
Definition 3.3. A linear operator $(B, D(B))$ on a Banach space $(\mathbb{X},\|\cdot\|)$ is dissipative if

$$
\|(\lambda-B) u\| \geq \lambda\|u\|
$$

for all $\lambda>0$ and all $u \in D(B)$.

One can refer to Section II.3 of [14] for more discussion of this concept.

Proposition 3.4. Assume (C1). Then the operator $(A, D(A))$ is dissipative on $\left(L^{1}\left(\mathbb{R}^{d}\right),\|\cdot\|_{1}\right)$.

Proof. Let $u \in D(A)$ and define $E^{ \pm}:=\left\{x \in \mathbb{R}^{d}: \pm u(x)>0\right\}$. Since $E^{ \pm} \subset \mathbb{R}^{d}$, $|u|= \pm u$ on $E^{ \pm}$, and $|(\lambda-A) u| \geq(\lambda-A)( \pm u)$,

$$
\|(\lambda-A) u\|_{1} \geq \int_{E^{+} \cup E^{-}}|(\lambda-A) u| \geq \int_{E^{+} \cup E^{-}}(\lambda-A)|u| .
$$

We claim that

$$
\int_{E^{ \pm}} A|u| \leq 0
$$

and hence the proposition follows since

$$
\|(\lambda-A) u\|_{1} \geq \int_{E^{+} \cup E^{-}} \lambda|u|=\lambda\|u\|_{1} .
$$

Now we prove claim (3.1) for the $E^{+}$case. If $E^{+} \neq \emptyset$, we can approximate it by sets $E_{\varepsilon_{n}}^{+}=\left\{x: u(x)>\varepsilon_{n}\right\}, 0<\varepsilon_{n} \rightarrow 0$, with $C^{1}$ boundaries. This can be done since by Sard's theorem and the implicit function theorem, $E_{\varepsilon}^{+}$has $C^{1}$ boundary for a.e. $0<\varepsilon<\max u^{+}$. Note that $E_{\varepsilon}^{ \pm} \subset E^{ \pm} \subset \operatorname{supp} u$ which is compact.

Then we write $A$ in divergence form, cf. (2.5),

$$
A \phi=\frac{1}{2} \operatorname{div}\left(a \nabla \phi+\left(\operatorname{div} a_{1}, \cdots, \operatorname{div} a_{d}\right)^{T} \phi-2 b \phi\right),
$$

and use the divergence theorem. We consider first the diffusion term,

$$
\int_{E_{\varepsilon}^{+}} \operatorname{div}(a \nabla u)=\int_{\partial E_{\varepsilon}^{+}} a \nabla u \cdot \mathbf{n},
$$

where $\mathbf{n}$ the exterior unit normal vector of $\partial E_{\varepsilon}^{+}$. Since $\partial E_{\varepsilon}^{+}$is an $\varepsilon$-level set of $u$ and $u$ is decreasing in the outward direction at $\partial E_{\varepsilon}^{+}, \nabla u=-\beta \mathbf{n}$ for some $\beta \geq 0$. Then since $a=\sigma \sigma^{T}$ is positive semi-definite

$$
a \nabla u \cdot \mathbf{n}=-\beta \mathbf{n}^{T} a \mathbf{n} \leq 0,
$$

and hence

$$
\int_{E_{\varepsilon}^{+}} \operatorname{div}(a \nabla u) \leq 0
$$

Next we estimate the convection part. Since $u=\varepsilon$ on $\partial E_{\varepsilon}^{+}$,

$$
\begin{aligned}
& \int_{E_{\varepsilon}^{+}} \operatorname{div}\left(\left(\operatorname{div} a_{1}, \cdots, \operatorname{div} a_{d}\right)^{T} u-2 b u\right)=\int_{\partial E_{\varepsilon}^{+}}\left(\left(\operatorname{div} a_{1}, \cdots, \operatorname{div} a_{d}\right)^{T}-2 b\right) u \cdot \mathbf{n} \\
& =\varepsilon \int_{\partial E_{\varepsilon}^{+}}\left(\left(\operatorname{div} a_{1}, \cdots, \operatorname{div} a_{d}\right)^{T}-2 b\right) \cdot \mathbf{n}=\varepsilon \int_{E_{\varepsilon}^{+}} \operatorname{div}\left(\left(\operatorname{div} a_{1}, \cdots, \operatorname{div} a_{d}\right)^{T}-2 b\right),
\end{aligned}
$$


which is bounded by $C \varepsilon$ for some $C=C(a, b, \operatorname{supp} u)$ since $a$ and $b$ are smooth and $u$ has compact support. Hence

$$
\int_{E_{\varepsilon}^{+}} A u \leq C \varepsilon
$$

Note that $\chi_{E_{\varepsilon}^{+}} \rightarrow \chi_{E^{+}}$a.e. Since $A u$ belongs to $L^{1}\left(\mathbb{R}^{d}\right)$, we may then use the dominated convergence theorem to pass to the limit in (3.2) as $\varepsilon \rightarrow 0^{+}$and obtain (3.1). The $E^{-}$case is similar and will be omitted. The proof is complete.

Now we proceed to prove Theorem 2.11. To do that we need the Chernoff Product Formula (e.g. Theorem III.5.2 in [14]):

Theorem 3.5. Let $\mathbb{X}$ be a Banach space, $V: \mathbb{R}_{+} \rightarrow \mathcal{L}(\mathbb{X})$ a function such that $V(0)=i d$ and $\left\|V^{n}(t)\right\| \leq M$ for all $t \geq 0$, all $n \in \mathbb{N}$, and some $M \geq 1$. Assume

$$
B u:=\lim _{t \rightarrow 0+} \frac{V(t) u-u}{t}
$$

exists for all $u \in D \subset \mathbb{X}$, where $D$ and $(\lambda-B) D$ are dense subspaces in $\mathbb{X}$ for some $\lambda>0$. Then the closure $\bar{B}$ of $B$ generates a bounded strongly continuous semigroup $\{T(t): t \geq 0\}$, given by

$$
T(t) u=\lim _{n \rightarrow+\infty} V^{n}(t / n) u,
$$

for all $u \in \mathbb{X}$ uniformly for $t \in[0, T]$.

This result does not require the approximation operators $V(t)$ to be a semigroup. This is important since the discrete path integration operators $\left\{\bar{P}_{\tau}\right\}$ are bounded on the Banach space $L^{1}\left(\mathbb{R}^{d}\right)$ by Proposition 2.9. But in contrast to their continuous counterpart, they no longer form a semigroup of operators ${ }^{4}$.

Proof of Theorem 2.11. We use Theorem 3.5 with $\mathbb{X}=L^{1}\left(\mathbb{R}^{d}\right), V(h)=\bar{P}_{h}, B=A$, and $D=D(A)$. Let us verify the conditions. By Proposition 2.10,

$$
A u=\lim _{h \rightarrow 0} \frac{\bar{P}_{h} u-u}{h}
$$

for all $u \in D(A)$. By the definition of $D(A)$ and Theorems 3.4 and 3.1, $A$ is densely defined, dissipative, and the closure $\bar{A}$ generates a contraction semigroup $P_{t}$ on $L^{1}\left(\mathbb{R}^{d}\right)$. Then by the Lumer-Phillips Theorem (e.g. Theorem II.3.15 in [14]), this implies that $(\lambda-A) D(A)$ is dense in $L^{1}\left(\mathbb{R}^{d}\right)$ for some $\lambda>0$.

Since the semigroup is strongly continuous by Lemma 3.2, we have verified all the conditions for Theorem 3.5. Hence hence can conclude that

$$
\left\|u(t, \cdot)-\bar{u}_{n}\right\|_{1}=\left\|P_{t} u_{0}-\bar{P}_{t / n}^{n} u_{0}\right\|_{1} \rightarrow 0,
$$

as $n \rightarrow \infty$ uniformly in $[0, T]$. The proof is complete.

\footnotetext{
${ }^{4}$ E.g. in Example 2.13, $\bar{P}_{\tau}^{2} \neq \bar{P}_{2 \tau}$ since

$$
\begin{aligned}
& \left(\bar{P}_{\tau}^{2} f\right)(y)=\int_{\mathbb{R}} \frac{f(x)}{\sqrt{4 \pi \tau \sigma^{2}\left(1+b \tau+b^{2} \tau^{2} / 2\right)}} \exp \left\{-\frac{\left(y-\left(1+2 b \tau+b^{2} \tau^{2}\right) x\right)^{2}}{4 \tau \sigma^{2}\left(1+b \tau+b^{2} \tau^{2} / 2\right)}\right\} d x, \\
& \left(\bar{P}_{2 \tau} f\right)(y)=\int_{\mathbb{R}} \frac{f(x)}{\sqrt{4 \pi \tau \sigma^{2}}} \exp \left\{-\frac{(y-(1+2 b \tau) x)^{2}}{4 \tau \sigma^{2}}\right\} d x .
\end{aligned}
$$
}


Now we prove Theorem 2.4, using among other things Theorem 2.11.

Proof of Theorem 2.4. We will show that (2.3) holds.

Assume first that $\phi \in C_{b}^{3}\left(\mathbb{R}^{d}\right)$. In view of the Euler-Maruyama scheme (2.8) corresponding to $Y_{t}$ with $\tau=\frac{t}{n}$, the PDF of its solution $X_{n}$ is $\bar{u}_{n}:=\bar{P}_{t / n}^{n} u_{0}$, and hence

$$
\mathbb{E} \phi\left(X_{n}\right)=\int_{\mathbb{R}^{d}} \phi(x) \bar{u}_{n}(x) d x \quad \text { for any } \quad \phi \in C_{b}^{0}\left(\mathbb{R}^{d}\right) .
$$

The right hand side is close to what we want, since by Theorem 2.11,

$$
\left|\int \phi \bar{u}_{n}-\int \phi u\right| \leq\|\phi\|_{\infty} \sup _{t \in[0, T]}\left\|u-\bar{u}_{n}\right\|_{1} \rightarrow 0 \quad \text { as } \quad n \rightarrow \infty .
$$

To see that the left hand side of (3.5) is also close to the sought after expression, we will use the weak convergence of the Euler-Maruyama method. Since our initial distribution $u_{0}$ is not assumed to have second moments, we need to introduce the following auxiliary SDE and Euler-Maruyama scheme for a fixed $x \in \mathbb{R}$ :

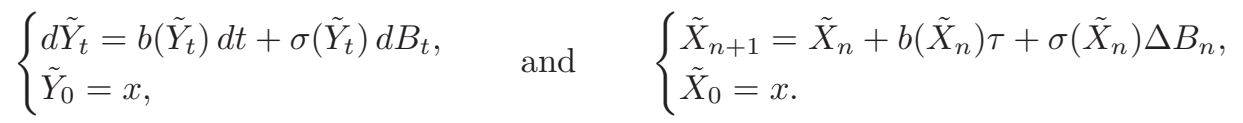

Both problems have unique (strong) solutions (Proposition 2.2), so we may define

$$
v(t, x):=\mathbb{E} \phi\left(\tilde{Y}_{t}\right) \quad \text { and } \quad v_{n}(x):=\mathbb{E} \phi\left(\tilde{X}_{n}\right) .
$$

By the strong Markov property of solutions of SDEs, cf. Theorem V.32 in [38],

$$
\mathbb{E} \phi\left(Y_{t}\right)=\mathbb{E}\left[\mathbb{E}\left(\phi\left(Y_{t}\right) \mid Y_{0}=Z\right)\right]=\mathbb{E}[v(t, Z)] .
$$

Similarly, since $\left\{X_{n}\right\}$ is a Markov chain,

$$
\mathbb{E} \phi\left(X_{n}\right)=\mathbb{E}\left[\mathbb{E}\left(\phi\left(X_{n}\right) \mid X_{0}=Z\right)\right]=\mathbb{E}\left[v_{n}(Z)\right] .
$$

Therefore

$$
\left|\mathbb{E} \phi\left(Y_{t}\right)-\mathbb{E} \phi\left(X_{n}\right)\right| \leq \mathbb{E}\left|v(t, Z)-v_{n}(Z)\right|=\int_{\mathbb{R}^{d}}\left|v(t, x)-v_{n}(x)\right| u_{0}(x) d x .
$$

The latter integrand is dominated by the integrable function $2\|\phi\|_{\infty}$, and by the weak convergence of the Euler-Maruyama scheme, cf. Theorem 14.1.5 in [24],

$$
\left|v(t, x)-v_{n}(x)\right| \rightarrow 0 \quad \text { as } \quad n \rightarrow \infty \quad \text { for every } x \in \mathbb{R}^{d} .
$$

Hence by (C3) and the dominated convergence theorem, it follows that

$$
\int_{\mathbb{R}^{d}}\left|v(t, x)-v_{n}(x)\right| u_{0}(x) d x \rightarrow 0 \quad \text { as } \quad n \rightarrow \infty .
$$

Note that the Theorem of [24] requires the initial probability measure to have finite second moments which is trivially met for the auxiliary problems above.

From all of the estimates above, we can the conclude that

$$
\left|\mathbb{E} \phi\left(Y_{t}\right)-\int \phi u\right| \leq\left|\mathbb{E} \phi\left(Y_{t}\right)-\mathbb{E} \phi\left(X_{n}\right)\right|+\left|\int \phi \bar{u}_{n}-\int \phi u\right| \rightarrow 0 \quad \text { as } n \rightarrow \infty,
$$

and hence that

$$
\mathbb{E} \phi\left(Y_{t}\right)=\int_{\mathbb{R}^{d}} \phi(x) u(t, x) d x \quad \text { for all } \quad \phi \in C_{b}^{3}\left(\mathbb{R}^{d}\right) .
$$


Now we assume $\phi \in C_{b}^{0}\left(\mathbb{R}^{d}\right)$ and conclude the proof by a regularization argument. Let $\rho_{\varepsilon} \in C_{c}^{\infty}, \varepsilon>0$, be the standard mollifier and $\phi_{\varepsilon}:=\rho_{\varepsilon} * \phi$. Then by e.g. Section 7.2 in [18], $\phi_{\varepsilon} \in C_{b}^{\infty}\left(\mathbb{R}^{d}\right),\left\|\phi_{\varepsilon}\right\|_{\infty} \leq\|\phi\|_{\infty}$, and $\phi_{\varepsilon} \rightarrow \phi$ a.e. Observe that

$$
\begin{aligned}
& \left|\int_{\mathbb{R}^{d}} \phi(x) u(t, x) d x-\mathbb{E} \phi\left(Y_{t}\right)\right| \\
& \leq\left|\int \phi u-\int \phi_{\varepsilon} u\right|+\left|\int \phi_{\varepsilon} u-\mathbb{E} \phi_{\varepsilon}\left(Y_{t}\right)\right|+\left|\mathbb{E} \phi_{\varepsilon}\left(Y_{t}\right)-\mathbb{E} \phi\left(Y_{t}\right)\right| .
\end{aligned}
$$

The second term is zero by our result for $\phi \in C_{b}^{3}\left(\mathbb{R}^{d}\right)$, and the two remaining terms tend to zero as $\varepsilon \rightarrow 0+$ by the dominated convergence theorem. The proof is complete.

\section{Proof of Proposition 2.10}

This section is devoted to the proof of Proposition 2.10. The key idea comes from the proof of Lemma 16 in [8], and we extend it to the case with unbounded coefficients.

Proof. Since $u \in C_{c}^{\infty}\left(\mathbb{R}^{d}\right)$, there is $R>0$ such that supp $u \subset B_{R}(0)$. The PDF after one-step Euler-Maruyama scheme is $\bar{P}_{\tau} u$. By Lemma 2.7,

$$
\begin{aligned}
& \mathcal{F}\left(\bar{P}_{\tau} u-u-\tau A u\right) \\
& =\int_{\mathbb{R}^{d}} u(x) e^{i \xi x}\left(\hat{\bar{k}}(\xi, x, \tau)-1-\tau\left(i b(x) \xi-\frac{1}{2} \xi^{T} a(x) \xi\right)\right) d x \\
& =\tau^{2} \iint_{0}^{1} u(x) e^{i \xi x}(1-\theta)\left(i b(x) \xi-\frac{1}{2} \xi^{T} a(x) \xi\right)^{2} e^{\theta \tau\left(i b(x) \xi-\frac{1}{2} \xi^{T} a(x) \xi\right)} d \theta d x .
\end{aligned}
$$

Without loss of generality, we assume $d=1$. Note that $D_{x}^{k} e^{i \xi x}=i^{k} \xi^{k} e^{i \xi x}$, we have

$$
\begin{aligned}
& \mathcal{F}\left(\bar{P}_{\tau} u-u-\tau A u\right) \\
& =\tau^{2} \int_{0}^{1}(1-\theta) \int_{\mathbb{R}} e^{i \xi x}\left[D_{x}^{2}\left(b(x) u(x) e^{\theta \tau\left(i b(x) \xi-\frac{1}{2} a(x) \xi^{2}\right)}\right)\right. \\
& \quad+D_{x}^{3}\left(a(x) b(x) u(x) e^{\theta \tau\left(i b(x) \xi-\frac{1}{2} a(x) \xi^{2}\right)}\right) \\
& \left.\quad+\frac{1}{4} D_{x}^{4}\left(a^{2}(x) u(x) e^{\theta \tau\left(i b(x) \xi-\frac{1}{2} a(x) \xi^{2}\right)}\right)\right] d x d \theta \\
& =\tau^{2} \int_{0}^{1}(1-\theta) \int_{\mathbb{R}} e^{i \xi x} e^{\theta \tau\left(i b(x) \xi-\frac{1}{2} a(x) \xi^{2}\right)} \sum_{m, n=0}^{4} \psi_{m, n}(x)(i \theta t \xi)^{m}\left(-\theta t \xi^{2}\right)^{n} d x d \theta
\end{aligned}
$$

where $\psi_{m, n} \in C_{c}(\mathbb{R})$ and for all $x \in \mathbb{R}$,

$$
\left|\psi_{m, n}(x)\right| \leq C_{m, n} \sum_{k=0}^{4}\left|D^{k} u(x)\right|,
$$

and the positive constant $C_{m, n}$ depends on $b, \sigma$, their derivatives up to fourth order, and $R$. 
Now we perform inverse Fourier transform and estimate it in $L^{1}$-norm

$$
\begin{aligned}
& \left\|\bar{P}_{\tau} u-u-\tau A u\right\|_{1} \\
& =\tau^{2} \int_{\mathbb{R}} \mid \int_{\mathbb{R}} \int_{0}^{1}(1-\theta) \int_{\mathbb{R}} e^{i \xi x} e^{\theta \tau\left(i b(x) \xi-\frac{1}{2} a(x) \xi^{2}\right)} \\
& \quad \cdot \sum_{m, n=0}^{4} \psi_{m, n}(x)(i \theta t \xi)^{m}\left(-\theta t \xi^{2}\right)^{n} d x d \theta d \xi \mid d y \\
& \leq \tau^{2} \int_{0}^{1} \int_{\mathbb{R}} \mid \int_{\mathbb{R}} \int_{\mathbb{R}} e^{i \xi(x-y)} e^{\theta \tau\left(i b(x) \xi-\frac{1}{2} a(x) \xi^{2}\right)} \\
& \quad \cdot \sum_{m, n=0}^{4} \psi_{m, n}(x)(i \theta t \xi)^{m}\left(-\theta t \xi^{2}\right)^{n} d x d \xi \mid d y d \theta \\
& =\tau^{2} \int_{0}^{1} \int_{\mathbb{R}} \mid \int_{\mathbb{R}} \int_{\mathbb{R}} e^{i \eta \frac{x-y}{\sqrt{\theta \tau}}} e^{i b(x) \sqrt{\theta \tau} \eta-\frac{1}{2} a(x) \eta^{2}} \\
& \quad \cdot \sum_{m, n=0}^{4} \psi_{m, n}(x)(i \sqrt{\theta \tau} \eta)^{m}\left(-\eta^{2}\right)^{n} \frac{d x}{\sqrt{\theta \tau}} d \eta \mid d y d \theta \\
& =\tau^{2} \int_{0}^{1} \int_{\mathbb{R}} \mid \int_{\mathbb{R}} \int_{\mathbb{R}} e^{i \eta z} e^{i b(y+\sqrt{\theta \tau} z) \sqrt{\theta \tau} \eta-\frac{1}{2} a(y+\sqrt{\theta \tau} z) \eta^{2}} \\
& \quad \sum_{m, n=0}^{4} \psi_{m, n}(y+\sqrt{\theta \tau} z)(i \sqrt{\theta \tau} \eta)^{m}\left(-\eta^{2}\right)^{n} d z d \eta \mid d y d \theta .
\end{aligned}
$$

After the above changes of variables, we continue the estimate

$$
\begin{aligned}
& \left\|\bar{P}_{\tau} u-u-\tau A u\right\|_{1} \\
& \leq \tau^{2} \int_{0}^{1} \int_{\mathbb{R}} \mid \int_{\mathbb{R}} \sum_{m, n=0}^{4} \psi_{m, n}(y+\sqrt{\theta \tau} z)(i \sqrt{\theta \tau})^{m}(-1)^{n} \\
& \cdot\left(\int_{\mathbb{R}} e^{i \eta z} \eta^{m+2 n} e^{i b(y+\sqrt{\theta \tau} z) \sqrt{\theta \tau} \eta-\frac{1}{2} a(y+\sqrt{\theta \tau} z) \eta^{2}} d \eta\right) d z \mid d y d \theta \\
& =\tau^{2} \int_{0}^{1} \int_{\mathbb{R}} \mid \int_{\mathbb{R}} \sum_{m, n=0}^{4} \psi_{m, n}(y+\sqrt{\theta \tau} z)(\sqrt{\theta \tau})^{m} \\
& \cdot\left(D_{v}^{m+2 n} \int_{\mathbb{R}} e^{i(v+b(y+\sqrt{\theta \tau} z) \sqrt{\theta \tau}) \eta} e^{-\frac{1}{2} a(y+\sqrt{\theta \tau} z) \eta^{2}} d \eta\right)_{v=z} d z \mid d y d \theta \\
& =\tau^{2} \int_{0}^{1} \int_{\mathbb{R}} \mid \int_{\mathbb{R}} \sum_{m, n=0}^{4} \psi_{m, n}(y+\sqrt{\theta \tau} z)(\sqrt{\theta \tau})^{m} \\
& \cdot\left(D_{v}^{m+2 n} \frac{1}{\sqrt{2 \pi a(y+\sqrt{\theta \tau} z)}} e^{-\frac{(v+b(y+\sqrt{\theta \tau} z) \sqrt{\theta \tau})^{2}}{2 a(y+\sqrt{\theta \tau} z)}}\right)_{v=z} d z \mid d y d \theta .
\end{aligned}
$$


Now there are no more oscillatory integrals, and therefore we can continue the estimate as follows

$$
\begin{aligned}
& \left\|\bar{P}_{\tau} u-u-\tau A u\right\|_{1} \\
& \leq \tau^{2} \sum_{m, n=0}^{4} \int_{0}^{1} \int_{\mathbb{R}} \int_{\mathbb{R}}\left|\psi_{m, n}(y+\sqrt{\theta \tau} z)\right| \\
& \cdot\left|\left(D_{v}^{m+2 n} \frac{1}{\sqrt{2 \pi a(y+\sqrt{\theta \tau} z)}} e^{-\frac{(v+b(y+\sqrt{\theta \tau} z) \sqrt{\theta \tau})^{2}}{2 a(y+\sqrt{\theta \tau} z)}}\right)_{v=z}\right| d y d z d \theta \\
& =\tau^{2} \sum_{m, n=0}^{4} \int_{0}^{1} \int_{\mathbb{R}} \int_{\mathbb{R}}\left|\psi_{m, n}(x)\left(D_{v}^{m+2 n} \frac{1}{\sqrt{2 \pi a(x)}} e^{-\frac{(v+b(x) \sqrt{\theta \tau})^{2}}{2 a(x)}}\right)_{v=z}\right| d x d z d \theta \\
& \leq \tau^{2} \sum_{m, n=0}^{4} \int_{0}^{1} \int_{\mathbb{R}} \int_{\mathbb{R}}\left|\psi_{m, n}(x)\right| \\
& \cdot \frac{C_{m, n}^{\prime}}{\sqrt{2 \pi a(x)}}\left(1+\frac{(z+\sqrt{\theta \tau} b(x))^{2(m+2 n)}}{a^{m+2 n}(x)}\right) e^{-\frac{\left(z+\sqrt{\theta \bar{\theta} b(x))^{2}}\right.}{2 a(x)}} d x d z d \theta \\
& \leq C_{1} \tau^{2} \sum_{m, n=0}^{4} \int_{0}^{1} \int_{\mathbb{R}}\left|\psi_{m, n}(x)\right| \\
& \cdot\left(\int_{\mathbb{R}} \frac{1}{\sqrt{2 \pi a(x)}}\left(1+\left(\frac{z+\sqrt{\theta \tau} b(x)}{\sqrt{a(x)}}\right)^{2(m+2 n)}\right) e^{-\frac{\left(z+\sqrt{\theta \bar{\tau} b(x))^{2}}\right.}{2 a(x)}} d z\right) d x d \theta .
\end{aligned}
$$

Finally we make a further change of variable $\tilde{z}=\frac{z+\sqrt{\theta \tau} b(x)}{\sqrt{a(x)}}$ and obtain

$$
\begin{aligned}
& \left\|\bar{P}_{\tau} u-u-\tau A u\right\|_{1} \\
& \leq C_{1} \tau^{2} \sum_{m, n=0}^{4} \int_{0}^{1} \int_{\mathbb{R}}\left|\psi_{m, n}(x)\right|\left(\int_{\mathbb{R}} \frac{1}{\sqrt{2 \pi}}\left(1+\tilde{z}^{2(m+2 n)}\right) e^{-\frac{\tilde{z}^{2}}{2}} d \tilde{z}\right) d x d \theta \\
& \leq C_{2} \tau^{2} \sum_{m, n=0}^{4}\left\|\psi_{m, n}\right\|_{1} \\
& \leq C \tau^{2} \sum_{k=0}^{4}\left\|D^{k} u\right\|_{1} .
\end{aligned}
$$

That is, $\left\|\frac{\bar{P}_{\tau} u-u}{\tau}-A u\right\|_{1} \leq C(b, \sigma, u) \tau$. The proof is complete.

Remark 4.1. From the above proof we see that the consistency estimate is uniform in the elliptic number $\alpha>0$ in condition (C2).

\section{Proof of Theorem 3.1}

To prove Theorem 3.1 we need first to show that operator $A$ and its adjoint satisfy certain properties, including being dissipative. 
Proposition 5.1. Assume (C1). Then the operators $A$ on $D(A):=C_{c}^{\infty}\left(\mathbb{R}^{d}\right)$ is densely defined, dissipative, and closable (possessing a closed extension) in $L^{1}\left(\mathbb{R}^{d}\right)$.

Remark 5.2. In view of Theorem 3.5 and Proposition 3.4 we only need $A$ to be closable. Alternatively we could have shown directly that $A$ is closed with respect to the operator norm $\|u\|_{A}:=\|u\|_{1}+\|A u\|_{1}$ as in Section 2 of [15].

Proof of Proposition 5.1. The operator $A$ is densely defined since $D(A)$ is dense in $L^{1}\left(\mathbb{R}^{d}\right)$, and it is dissipative by Proposition 3.4. Hence it is closable by Proposition II.3.14 in [14].

On the dual space of $\left(L^{1}\left(\mathbb{R}^{d}\right),\|\cdot\|_{1}\right),\left(L^{\infty}\left(\mathbb{R}^{d}\right),\|\cdot\|_{\infty}\right)$, the adjoint operator of $A$ with domain $D(A)=C_{c}^{\infty}\left(\mathbb{R}^{d}\right)$ is given by the definition below.

Definition 5.3. The adjoint of $A$ is an operator $A^{*}: D\left(A^{*}\right) \rightarrow L^{\infty}\left(\mathbb{R}^{d}\right)$ defined as

$$
\int_{\mathbb{R}^{d}} u A^{*} f=\int_{\mathbb{R}^{d}} f A u \quad \text { for all } \quad f \in D\left(A^{*}\right) \text { and } u \in D(A),
$$

where $D\left(A^{*}\right)=\left\{f \in L^{\infty}: \exists g \in L^{\infty}\right.$ such that $\int f A u=\int g u$ for all $\left.u \in D(A)\right\}$.

Remark 5.4. By integration by parts in (2.5), $A^{*} f$ is given by (2.2) for any $f \in$ $C_{b}^{2}\left(\mathbb{R}^{d}\right)$. Moreover $f \in D\left(A^{*}\right)$ if and only if there exists a $g \in L^{\infty}\left(\mathbb{R}^{d}\right)$ such that

$$
A^{*} f=g \quad \text { in } \quad \mathcal{D}^{\prime} \text { (in distributions). }
$$

Proposition 5.5. Assume (C1) and (C2). Then the adjoint operator $\left(A^{*}, D\left(A^{*}\right)\right)$ is dissipative on $\left(L^{\infty}\left(\mathbb{R}^{d}\right),\|\cdot\|_{\infty}\right)$.

The proof is quite technical, so we postpone it to the end of this section. Now we can employ the following result to prove that $A$ generates a contraction semigroup.

Proof of Theorem 3.1. Note that $A$ with $D(A)=C_{c}^{\infty}\left(\mathbb{R}^{d}\right)$ is a bounded densely defined operator on $L^{1}\left(\mathbb{R}^{d}\right)$, and that both $A$ and its adjoint $A^{*}$ are dissipative by Propositions 5.1 and 5.5. Hence the closure $\bar{A}$ generates a contraction semigroup on $\left(L^{1}\left(\mathbb{R}^{d}\right),\|\cdot\|_{1}\right)$ by Corollary II.3.17 in [14]. The proof is complete.

In the rest of this section we prove Proposition 5.5.

Lemma 5.6. Assume (C1) and (C2), then

$$
D\left(A^{*}\right) \subset\left\{f \in C^{1}\left(\mathbb{R}^{d}\right) \cap L^{\infty}\left(\mathbb{R}^{d}\right): \nabla^{2} f \in \bigcap_{1<p<\infty} L_{l o c}^{p}\left(\mathbb{R}^{d}\right)\right\} .
$$

Remark 5.7. Under different assumptions, similar characterisations of the maximal domain can be found in Chapter 3 of [32] and Section 3 of [15].

Proof. For any $f \in D\left(A^{*}\right)$, there exists $g \in L^{\infty}\left(\mathbb{R}^{d}\right)$ such that (5.2) holds by Remark 5.4. By the regularity of the coefficients (see (C1)), we rewrite (5.1) as

$$
\begin{aligned}
& \int f \sum_{i, j=1}^{d} \partial_{i}\left(\frac{1}{2} a_{i j} \partial_{j} u\right) \\
& =\int\left(g+f \sum_{i=1}^{d} \partial_{i} b_{i}-\frac{1}{2} f \sum_{i, j=1}^{d} \partial_{i} \partial_{j} a_{i j}\right) u+f \sum_{i=1}^{d}\left(b_{i}-\frac{1}{2} \sum_{j=1}^{d} \partial_{j} a_{i j}\right) \partial_{i} u
\end{aligned}
$$


On the right hand side, the coefficients of $u$ and $\partial_{i} u$ belong to $L_{l o c}^{p}\left(\mathbb{R}^{d}\right)$ for all $1<p<\infty$. We can therefore apply Theorem 1.5 in [42] to show that $f \in W_{l o c}^{1, p}\left(\mathbb{R}^{d}\right)$ for all $1<p<\infty$. With this extra regularity and integration by parts,

$$
\int f \sum_{i, j=1}^{d} \partial_{i}\left(\frac{1}{2} a_{i j} \partial_{j} u\right)=\int\left(g+\frac{1}{2} \sum_{i, j=1}^{d} \partial_{i} f \partial_{j} a_{i j}-\sum_{i=1}^{d} b_{i} \partial_{i} f\right) u .
$$

On the right hand side, the coefficients of $u$ belong to $L_{l o c}^{p}\left(\mathbb{R}^{d}\right)$ for all $1<p<\infty$ while the coefficient of $\partial_{i} u$ is zero. By Proposition 1.1 in [42] we then have that $f \in W_{l o c}^{2, p}\left(\mathbb{R}^{d}\right)$ for all $1<p<\infty$.

Finally, by taking $p>d$ in the Sobolev embedding theorem, see e.g. Theorem 7.10 in [18], we find that $\nabla f \in C\left(\mathbb{R}^{d}\right)$. The proof is complete.

The next lemma is crucial.

Lemma 5.8. Assume $f \in D\left(A^{*}\right)$ and $m:=\sup _{\mathbb{R}^{d}} f<\infty$. Then there exists a sequence $\left\{x_{n}\right\} \subset \mathbb{R}^{d}$ such that

$$
\lim _{n} f\left(x_{n}\right)=m, \quad \lim _{n}\left(1+\left|x_{n}\right|\right)\left|\nabla f\left(x_{n}\right)\right|=0, \quad \text { and } \quad \lim _{n} \frac{\left|x_{n}\right|^{2}}{n}=0 .
$$

Moreover, $\nabla^{2} f\left(x_{n}\right)$ exists and

$$
\nabla^{2} f\left(x_{n}\right) \leq \frac{3}{n} I_{d}
$$

where $I_{d}$ is the identity matrix on $\mathbb{R}^{d}$.

Since the second derivatives are only defined almost everywhere, we need the socalled Bony maximum principle $[6,30]$ to prove the above lemma. We quote the version given in Proposition 1.2.12 in [17].

Lemma 5.9. Suppose $p \geq d$ and a function $w \in W_{l o c}^{2, p}\left(\mathbb{R}^{d}\right)$ achieves a local maximum at point $x_{0}$, then for all $\xi \in \mathbb{R}^{d}$,

$$
\lim _{r \rightarrow 0}\left(\underset{B_{r}\left(x_{0}\right)}{\operatorname{essinf}} \sum_{i, j=1}^{d} \xi_{i}\left(\partial_{i} \partial_{j} w\right) \xi_{j}\right) \leq 0,
$$

where $B_{r}\left(x_{0}\right)$ denotes the ball of radius $r$ centered at $x_{0}$.

The key idea of the following proof now comes from Lemma 2.3 in [23].

Proof of Lemma 5.8. For each $n \in \mathbb{N}$, we define

$$
g_{n}(x):=f(x)-\frac{|x|^{2}}{n} .
$$

Hence $g_{n}$ is bounded from above and tends to $-\infty$ when $|x| \rightarrow \infty$. Therefore there exists $x_{n}^{\prime}$ for each $g_{n}$ such that

$$
g_{n}\left(x_{n}^{\prime}\right)=\max _{\mathbb{R}^{d}} g_{n}=: m_{n} .
$$

Now for arbitrary $\epsilon>0$, we can find first $x_{\epsilon} \in \mathbb{R}^{d}$ and then $N \in \mathbb{N}$ such that

$$
f\left(x_{\epsilon}\right)>m-\epsilon \quad \text { and } \quad \frac{\left|x_{\epsilon}\right|^{2}}{n}<\epsilon \quad \text { for all } n>N .
$$


Hence for all $n>N$,

$$
\begin{aligned}
m & \geq m_{n}=f\left(x_{n}^{\prime}\right)-\frac{\left|x_{n}^{\prime}\right|^{2}}{n}=\max _{\mathbb{R}^{d}} g_{n} \\
& \geq g_{n}\left(x_{\epsilon}\right)=f\left(x_{\epsilon}\right)-\frac{\left|x_{\epsilon}\right|^{2}}{n} \\
& \geq m-2 \epsilon .
\end{aligned}
$$

Therefore $m_{n} \rightarrow m$ as $n \rightarrow \infty$, and hence $f\left(x_{n}^{\prime}\right) \rightarrow m$ and $\frac{\left|x_{n}^{\prime}\right|^{2}}{n} \rightarrow 0$ as $n \rightarrow \infty$.

Next we analyse the derivatives. By definitions of $x_{n}^{\prime}$ and $g_{n}$,

$$
0=\nabla g_{n}\left(x_{n}^{\prime}\right)=\nabla f\left(x_{n}^{\prime}\right)-\frac{2}{n} x_{n}^{\prime} .
$$

Hence, as $n \rightarrow \infty$,

$$
\left(1+\left|x_{n}^{\prime}\right|\right)\left|\nabla f\left(x_{n}^{\prime}\right)\right|=\left(1+\left|x_{n}^{\prime}\right|\right) \frac{2}{n}\left|x_{n}^{\prime}\right| \leq \frac{1}{n}\left(1+3\left|x_{n}^{\prime}\right|^{2}\right) \rightarrow 0 .
$$

Finally for the second derivatives,

$$
\nabla^{2} g_{n}(x)=\nabla^{2} f(x)-\frac{2}{n} I_{d}, \text { a.e. }
$$

Since $x_{n}^{\prime}$ is maximum point for $g_{n}$, by Proposition 5.9,

$$
\lim _{r \rightarrow 0}\left(\underset{B_{r}\left(x_{n}^{\prime}\right)}{\operatorname{essinf}} \nabla^{2} g_{n}\right) \leq 0, \quad \text { and hence } \quad \lim _{r \rightarrow 0}\left(\underset{B_{r}\left(x_{n}^{\prime}\right)}{\operatorname{essinf}} \nabla^{2} f\right) \leq \frac{2}{n} I_{d} .
$$

By the definition of the infimum and regularity of $f$, we can find another sequence $\left\{x_{n}\right\}$ such that $\nabla^{2} f\left(x_{n}\right)$ is defined, $\left|x_{n}-x_{n}^{\prime}\right|<1 / n$, and

$$
\nabla^{2} f\left(x_{n}\right) \leq \frac{3}{n} I_{d}
$$

Using that $f \in C^{1}$, we can take a further subsequence such that also

$$
\left|f\left(x_{n}\right)-f\left(x_{n}^{\prime}\right)\right|+\left|\nabla f\left(x_{n}\right)-\nabla f\left(x_{n}^{\prime}\right)\right|<\frac{1}{n} .
$$

Combining all the above estimates, we can conclude that

$$
\lim _{n} f\left(x_{n}\right)=m, \quad \lim _{n}\left(1+\left|x_{n}\right|\right)\left|\nabla f\left(x_{n}\right)\right|=0, \quad \lim _{n} \frac{\left|x_{n}\right|^{2}}{n}=0,
$$

and $\nabla^{2} f\left(x_{n}\right) \leq \frac{3}{n} I_{d}$. The proof is complete.

Proof of Proposition 5.5. Let $f \in D\left(A^{*}\right)$. We may assume that $m:=\|f\|_{\infty}=$ $\sup _{\mathbb{R}^{d}} f$. The case $m=-\inf _{\mathbb{R}^{d}} f$ follows in a similar way and is omitted.

Note that by $(2.2), a_{i j}(x)=\sum_{k=1}^{n} \sigma_{i k}(x) \sigma_{j k}$ and $\sigma=\left(\sigma_{1}, \cdots, \sigma_{n}\right)$, and then

$$
A^{*} f(x)=\sum_{i=1}^{d} b_{i}(x) \partial_{i} f(x)+\frac{1}{2} \sum_{k=1}^{n} \sigma_{k}^{T}(x) \nabla^{2} f(x) \sigma_{k}(x) .
$$

Let $\left\{x_{n}\right\}$ be the sequence corresponding to $f$ given by Lemma 5.8. From the proof it follows that for any fixed $\lambda>0$, we can always take

$$
\left\{x_{n}\right\} \subset\left\{x \in \mathbb{R}^{d}:\left|\lambda f(x)-A^{*} f(x)\right| \leq\left\|\lambda f-A^{*} f\right\|_{\infty}\right\},
$$


because the complement of the latter set has zero Lebesgue measure in $\mathbb{R}^{d}$. By (C1) and Lemma 5.8,

$$
\lim _{n} f\left(x_{n}\right)=m, \quad \lim _{n}\left|b_{i}\left(x_{n}\right) \partial_{i} f\left(x_{n}\right)\right| \leq \lim _{n} K\left(1+\left|x_{n}\right|\right)\left|\nabla f\left(x_{n}\right)\right|=0,
$$

and

$$
\limsup _{n} \sigma_{k}^{T}\left(x_{n}\right) \nabla^{2} f\left(x_{n}\right) \sigma_{k}\left(x_{n}\right) \leq \lim _{n} \frac{3}{n}\left|\sigma_{k}\left(x_{n}\right)\right|^{2} \leq \lim _{n} \frac{3}{n} K\left(1+\left|x_{n}\right|^{2}\right)=0 .
$$

Hence,

$$
\limsup _{n}\left(-A^{*} f\left(x_{n}\right)\right) \geq 0
$$

and then

$$
\lambda\|f\|_{\infty}=\lambda \lim _{n} f\left(x_{n}\right) \leq \limsup _{n}\left(\lambda f\left(x_{n}\right)-A^{*} f\left(x_{n}\right)\right) \leq\left\|\left(\lambda-A^{*}\right) f\right\|_{\infty} .
$$

Since $f \in D\left(A^{*}\right)$ and $\lambda>0$ are both arbitrary, $A^{*}$ is a dissipative operator, and the proof is complete.

\section{Appendix A. Weak Convergence and Convergence of PDFs}

Now we demonstrate the relationship between $L^{1}$-convergence of PDFs and weak convergence of processes.

Proposition A.1. Assume $X_{n}$ and $X$ are random variables with PDFs $u_{n}$ and $u$ satisfying

$$
\lim _{n \rightarrow \infty}\left\|u_{n}-u\right\|_{1}=0
$$

Then $X_{n}$ converges weakly to $X$,

$$
\lim _{n \rightarrow \infty}\left|\mathbb{E} \phi\left(X_{n}\right)-\mathbb{E} \phi(X)\right|=0 \quad \text { for all } \quad \phi \in C_{b}^{0}\left(\mathbb{R}^{d}\right) .
$$

Proof. $\left|\mathbb{E} \phi\left(X_{n}\right)-\mathbb{E} \phi(X)\right|=\left|\int_{\mathbb{R}^{d}} \phi(x)\left(u_{n}-u\right)(x) d x\right| \leq\|\phi\|_{\infty}\left\|\bar{u}_{n}-u\right\|_{1}$.

The opposite is not true in general as the following example shows.

Example A.2. For any $n \in \mathbb{N}$, we define

$$
v_{n}(x)=(1+\sin (2 n \pi x)) \chi_{[0,1]}(x) .
$$

Evidently, $v_{n} \geq 0$ and $\int_{\mathbb{R}} v_{n}=1$, and hence $v_{n}$ is a PDF.

For any $n \in \mathbb{N}$, we take $X_{n}$ to be a random variable with PDF $v_{n}$. By the RiemannLebesgue Lemma, $X_{n}$ converges weakly to a random variable $X$ with PDF

$$
v(x) \equiv \chi_{[0,1]}(x) .
$$

However, $v_{n}$ does not converge to $v$ in $L^{1}$ since

$$
\left\|v_{n}-v\right\|_{1}:=\int_{\mathbb{R}}\left|v_{n}-v\right|=\int_{0}^{1}|\sin (2 n \pi x)| d x \equiv \frac{2}{\pi}>0 .
$$




\section{Appendix B. Proof of Proposition 2.14}

Fix $t \in[0, T]$ and define $\tau=t / n$ for $n>|b t|$. Without loss of generality, we assume that $b \neq 0$. We start with deriving an expression for the iteration of operators $\bar{P}_{\tau}^{n}$.

From the scheme (2.14) and an iteration, we find that

$$
X_{n}=(1+b \tau)^{n} X_{0}+\sigma \sum_{k=0}^{n-1}(1+b \tau)^{k} \Delta B_{n-k} .
$$

Let $\psi_{n}(\xi)$ and $\phi(\xi)=e^{-\frac{1}{2} \xi^{2}}$ be the characteristic functions of $X_{n}$ and an $N(0,1)$ Gaussian variable, respectively. To simplify the notations, let $C=C\left(b, \sigma, T, u_{0}\right)>0$ denote various positive constants depending only on $b, \sigma, T$, and $u_{0}$, and define

$$
\alpha:=e^{b t}, \quad \alpha_{n}:=(1+b \tau)^{n} ; \quad \beta^{2}:=\frac{\sigma^{2}}{b}\left(e^{2 b t}-1\right), \quad \beta_{n}^{2}:=\frac{\sigma^{2}}{b} \frac{2\left((1+b \tau)^{2 n}-1\right)}{2+b \tau} .
$$

By the independence of the increments, the definition of $\Delta B_{k}$, and summation of exponents,

$$
\psi_{n}(\xi)=\psi_{0}\left(\alpha_{n} \xi\right) \prod_{k=0}^{n-1} \phi\left(\alpha_{k} \sqrt{\tau} \sigma \xi\right)=\psi_{0}\left(\alpha_{n} \xi\right) e^{-\frac{1}{4} \beta_{n}^{2} \xi^{2}} .
$$

Note that $\psi_{0}(\xi)=\sqrt{2 \pi} \hat{u}_{0}(\xi)$ and $\psi_{n}(\xi)=\sqrt{2 \pi} \hat{\bar{u}}_{n}(\xi)$. Taking the inverse Fourier transform, and after elementary computations using convolution, scaling, and change of variables, we find that

$$
\begin{aligned}
\left(\bar{P}_{\tau}^{n} u_{0}\right)(y) & =\bar{u}_{n}(y)=\frac{1}{\sqrt{2 \pi}} \frac{u_{0}\left(\cdot / \alpha_{n}\right)}{\alpha_{n}} *\left(\frac{1}{\sqrt{\beta_{n}^{2} / 2}} e^{-\frac{2^{2}}{\beta_{n}^{2}}}\right)(y) \\
& =\int_{\mathbb{R}} \frac{u_{0}(x)}{\sqrt{\pi \beta_{n}^{2}}} \exp \left\{-\frac{\left(y-\alpha_{n} x\right)^{2}}{\beta_{n}^{2}}\right\} d x .
\end{aligned}
$$

Now we estimate the $L^{1}$-norm of the difference of the exact and the approximate densities, $\left\|\left(\bar{P}_{\tau}^{n}-P_{t}\right) u_{0}\right\|_{L^{1}}$. Recall (2.13) and decompose

$$
\begin{aligned}
\bar{P}_{\tau}^{n} u_{0}(y)-P_{t} u_{0}(y) & \int_{\mathbb{R}} \frac{u_{0}(x)}{\sqrt{\pi \beta_{n}^{2}}} \exp \left\{-\frac{\left(y-\alpha_{n} x\right)^{2}}{\beta_{n}^{2}}\right\} d x-\int_{\mathbb{R}} \frac{u_{0}(x)}{\sqrt{\pi \beta^{2}}} \exp \left\{-\frac{(y-\alpha x)^{2}}{\beta^{2}}\right\} d x \\
= & \beta_{n}\left(\frac{1}{\beta_{n}}-\frac{1}{\beta}\right) \int_{\mathbb{R}} \frac{u_{0}(x)}{\sqrt{\pi \beta_{n}^{2}}} \exp \left\{-\frac{\left(y-\alpha_{n} x\right)^{2}}{\beta_{n}^{2}}\right\} d x \\
& +\int_{\mathbb{R}} \frac{u_{0}(x)}{\sqrt{\pi \beta^{2}}}\left(\exp \left\{-\frac{\left(y-\alpha_{n} x\right)^{2}}{\beta_{n}^{2}}\right\}-\exp \left\{-\frac{\left(y-\alpha_{n} x\right)^{2}}{\beta^{2}}\right\}\right) d x \\
& +\int_{\mathbb{R}} \frac{u_{0}(x)}{\sqrt{\pi \beta^{2}}}\left(\exp \left\{-\frac{\left(y-\alpha_{n} x\right)^{2}}{\beta^{2}}\right\}-\exp \left\{-\frac{(y-\alpha x)^{2}}{\beta^{2}}\right\}\right) d x \\
= & : E_{1}+E_{2}+E_{3} .
\end{aligned}
$$

We start with some observations. Let $a \in \mathbb{R}$. By l'Hôpital's rule and a Taylor expansion of $\ln (1+a x)$ about $x=0$,

$$
\lim _{x \rightarrow 0} \frac{1}{x}\left(e^{a}-(1+a x)^{\frac{1}{x}}\right)=\frac{a^{2}}{2} e^{a}
$$


and hence there is a locally bounded function $\tilde{C}(a)>0$ such that

$$
\left|e^{a}-\left(1+\frac{a}{n}\right)^{n}\right| \leq \tilde{C}(a) \frac{a^{2}}{n} .
$$

Now for $E_{1}$, obviously $\left\|\int_{\mathbb{R}} \frac{u_{0}(x)}{\sqrt{\pi \beta_{n}^{2}}} \exp \left\{-\frac{\left(\cdot-\alpha_{n} x\right)^{2}}{\beta_{n}^{2}}\right\} d x\right\|_{1} \leq\left\|u_{0}\right\|_{1}$. We turn to analyse $\beta_{n}\left(\frac{1}{\beta_{n}}-\frac{1}{\beta}\right)$. Since

$$
\left|e^{x}-1\right| \geq \begin{cases}\frac{|x|}{2}, & -1 \leq x, \\ \frac{1}{2}, & x \leq-1,\end{cases}
$$

it follows that

$$
\beta^{2}:=\frac{\sigma^{2}}{b}\left(e^{2 b t}-1\right) \geq \begin{cases}\sigma^{2} t, & -1 \leq 2 b t \\ \frac{\sigma^{2}}{2|b|}, & 2 b t \leq-1\end{cases}
$$

In other words,

$$
\frac{1}{\beta} \leq \frac{1 \vee \sqrt{2|b| t}}{\sqrt{\sigma^{2} t}} \text { for all } t>0
$$

Moreover

$$
\beta^{2}-\beta_{n}^{2}=\frac{\sigma^{2}}{b}\left(e^{2 b t}-(1+b t / n)^{2 n}\right)+\beta_{n}^{2} \frac{b t / n}{2+b t / n} .
$$

Hence combining (B.4) and (B.1), we get

$$
\left|\beta_{n}\left(\frac{1}{\beta_{n}}-\frac{1}{\beta}\right)\right|=\left|\frac{\beta^{2}-\beta_{n}^{2}}{\beta\left(\beta+\beta_{n}\right)}\right| \leq\left|\frac{\beta^{2}-\beta_{n}^{2}}{\beta^{2}}\right| \leq \frac{\tilde{C}(2 b t) 4 b^{2} t^{2}}{n} \frac{\sigma^{2}}{2 t}=\frac{C t}{n} .
$$

Therefore, $\left\|E_{1}\right\|_{1} \leq C t / n$.

Next, we estimate $E_{2}$. By a change of variable and the commuting property of convolutions, we have

$$
\begin{aligned}
E_{2} & =\frac{1}{\alpha_{n}} \int_{\mathbb{R}} \frac{u_{0}\left(\frac{y-x}{\alpha_{n}}\right)}{\sqrt{\pi \beta^{2}}}\left(e^{-\frac{x^{2}}{\beta_{n}^{2}}}-e^{-\frac{x^{2}}{\beta^{2}}}\right) d x \\
& =\frac{1}{\alpha_{n}} \int_{\mathbb{R}} \frac{u_{0}\left(\frac{y-\beta x}{\alpha_{n}}\right)}{\sqrt{\pi}} e^{-x^{2}}\left(e^{-\frac{\beta_{n}^{2}-\beta^{2}}{\beta_{n}^{2}} x^{2}}-1\right) d x
\end{aligned}
$$

First we observe with (B.1) and (B.2) that

$$
\begin{aligned}
\beta_{n}^{2} & \geq \frac{2 \sigma^{2}}{3|b|}\left|1-(1+b \tau)^{2 n}\right| \geq \frac{2 \sigma^{2}}{3|b|}\left|1-e^{2 b t}\right|-\left|e^{2 b t}-(1+b \tau)^{2 n}\right| \\
& \geq \frac{\sigma^{2}}{3}\left(2 t \wedge \frac{1}{|b|}\right)-\frac{C t^{2}}{n} \geq \frac{\sigma^{2}}{6}\left(2 t \wedge \frac{1}{|b|}\right) \quad \text { for } \quad n>4 C T(1 \vee|b| T) .
\end{aligned}
$$

Hence again from (B.4) and (B.1) we have $\left|\frac{\beta_{n}^{2}-\beta^{2}}{\beta_{n}^{2}} x^{2}\right| \leq \frac{C t}{n} x^{2}$. Therefore

$$
e^{-\frac{C t}{n} x^{2}}-1 \leq e^{\frac{\beta_{n}^{2}-\beta^{2}}{\beta_{n}^{2}} x^{2}}-1 \leq e^{\frac{C t}{n} x^{2}}-1 .
$$


So $\left|e^{\frac{\beta_{n}^{2}-\beta^{2}}{\beta_{n}^{2}} x^{2}}-1\right| \leq\left(1-e^{-\frac{C t}{n} x^{2}}\right) \vee\left(e^{\frac{C t}{n} x^{2}}-1\right)=e^{\frac{C t}{n} x^{2}}-1$. Now we can use Fubini's theorem to conclude that

$$
\begin{aligned}
\left\|E_{2}\right\|_{1} & \leq \frac{1}{\alpha_{n}} \iint\left|\frac{u_{0}\left(\frac{y-\beta x}{\alpha_{n}}\right)}{\sqrt{\pi}} e^{-x^{2}}\left(e^{-\frac{\beta_{n}^{2}-\beta^{2}}{\beta_{n}^{2}} x^{2}}-1\right)\right| d x d y \\
& \leq\left\|u_{0}\right\|_{1} \frac{1}{\sqrt{\pi}} \int_{\mathbb{R}} e^{-x^{2}}\left(e^{\frac{C t}{n} x^{2}}-1\right) d x \\
& =\left\|u_{0}\right\|_{1}\left((1-C t / n)^{-1 / 2}-1\right) \\
& \leq \frac{C t}{n} \quad \text { for } n \text { large enough. }
\end{aligned}
$$

It remains to analyse $E_{3}$, and here the computations will differ according to the assumptions on $u_{0}$. We first assume that $\int_{\mathbb{R}}\left|x u_{0}(x)\right| d x<\infty$. Denote $\gamma_{n}:=\frac{\alpha-\alpha_{n}}{\beta}$. Combining (B.1) and (B.3), it follows that

$$
\left|\gamma_{n}\right| \leq \frac{\tilde{C}(b t) b^{2} t^{2}}{n} \frac{(1 \vee \sqrt{2|b| t})}{\sqrt{\sigma^{2} t}} \leq \frac{C t^{3 / 2}}{n}
$$

Therefore by a change of variable $y \mapsto y+\theta \gamma_{n} x$, we have

$$
\begin{aligned}
\left\|E_{3}\right\|_{1} & \leq \iint\left|\frac{u_{0}(x)}{\sqrt{\pi \beta^{2}}}\left(\exp \left\{-\frac{\left(y-\alpha_{n} x\right)^{2}}{\beta^{2}}\right\}-\exp \left\{-\frac{(y-\alpha x)^{2}}{\beta^{2}}\right\}\right)\right| d x d y \\
& =\iint\left|\frac{u_{0}(x)}{\sqrt{\pi}}\left(e^{-\left(y+\gamma_{n} x\right)^{2}}-e^{-y^{2}}\right)\right| d y d x \\
& =\iint \frac{\left|u_{0}(x)\right|}{\sqrt{\pi}}\left|\int_{0}^{1} \gamma_{n} x 2\left(y+\theta \gamma_{n} x\right) e^{-\left(y+\theta \gamma_{n} x\right)^{2}} d \theta\right| d y d x \\
& \leq \frac{2\left|\gamma_{n}\right|}{\sqrt{\pi}} \int_{\mathbb{R}}\left|x u_{0}(x)\right| d x \int_{\mathbb{R}}|z| e^{-z^{2}} d z \\
& \leq \frac{C t^{3 / 2}}{n} \quad \text { for } n \text { large enough. }
\end{aligned}
$$

For the other two cases, we write

$$
\begin{aligned}
E_{3}= & \int_{\mathbb{R}}\left(\frac{1}{\alpha_{n}} u_{0}\left(\frac{x}{\alpha_{n}}\right)-\frac{1}{\alpha} u_{0}\left(\frac{x}{\alpha}\right)\right) \frac{1}{\sqrt{\pi \beta^{2}}} e^{-\frac{(y-x)^{2}}{\beta^{2}}} d x \\
= & \left(\frac{1}{\alpha_{n}}-\frac{1}{\alpha}\right) \int_{\mathbb{R}} u_{0}\left(\frac{x}{\alpha_{n}}\right) \frac{1}{\sqrt{\pi \beta^{2}}} e^{-\frac{(y-x)^{2}}{\beta^{2}}} d x \\
& +\frac{1}{\alpha} \int_{\mathbb{R}}\left(u_{0}\left(\frac{x}{\alpha_{n}}\right)-u_{0}\left(\frac{x}{\alpha}\right)\right) \frac{1}{\sqrt{\pi \beta^{2}}} e^{-\frac{(y-x)^{2}}{\beta^{2}}} d x \\
= & : E_{3}^{\prime}+\frac{1}{\alpha} E_{3}^{\prime \prime} .
\end{aligned}
$$

Note that $\alpha_{n} \rightarrow \alpha>0$ and hence $0<\alpha / 2 \leq \alpha_{n} \leq 2 \alpha$ for large $n$. Since $\lim _{n} \alpha_{n}=$ $\alpha$, and $\alpha, \alpha_{n}$ are bounded away from 0. Combining (B.1), it follows that

$$
\left|\frac{1}{\alpha}-\frac{1}{\alpha_{n}}\right|=\left|\frac{e^{b t}-(1+b t / n)^{n}}{\alpha \alpha_{n}}\right| \leq \frac{1}{\alpha \alpha_{n}} \frac{\tilde{C}(b t) b^{2} t^{2}}{n} \leq \frac{C t^{2}}{n} .
$$


Hence $\left\|E_{3}^{\prime}\right\|_{1} \leq \frac{C t^{2}}{n}$ for large $n$.

Regarding to $E_{3}^{\prime \prime}$, we assume now $\int_{\mathbb{R}}\left|x u_{0}^{\prime}(x)\right| d x<\infty$. Then similarly

$$
\begin{aligned}
\left\|E_{3}^{\prime \prime}\right\|_{1} & \leq \int_{\mathbb{R}}\left|u_{0}\left(\frac{x}{\alpha}\right)-u_{0}\left(\frac{x}{\alpha_{n}}\right)\right| d x \\
& \leq \int_{\mathbb{R}} \int_{0}^{1}\left|\left(\frac{1}{\alpha_{n}}-\frac{1}{\alpha}\right) x u_{0}^{\prime}\left(\frac{x}{\alpha}+\theta\left(\frac{1}{\alpha_{n}}-\frac{1}{\alpha}\right) x\right)\right| d \theta d x \\
& \leq C\left|\frac{1}{\alpha_{n}}-\frac{1}{\alpha}\right| \int_{\mathbb{R}}\left|x u_{0}^{\prime}(x)\right| d x \leq \frac{C t^{2}}{n} .
\end{aligned}
$$

Finally we consider the most general case $u_{0} \in L^{1}(\mathbb{R})$ only. We will prove a uniform convergence to zero without any rate. For any $\varepsilon>0$, there exist $R>0$ and $N_{1}>0$ such that

$$
\int_{|x|>R}\left|u_{0}\left(\frac{x}{\alpha}\right)\right| d x<\frac{\varepsilon}{3} \quad \text { and } \quad \int_{|x|>R}\left|u_{0}\left(\frac{x}{\alpha_{n}}\right)\right| d x<\frac{\varepsilon}{3} \quad \text { for all } n>N_{1} .
$$

Next, since translation is continuous in $L^{1}$, i.e. $\lim _{h \rightarrow 0} \int_{\mathbb{R}}|w(x)-w(x+h)| d x=0$ for any $w \in L^{1}(\mathbb{R})$, and $h_{n}(x):=\left(\frac{1}{\alpha_{n}}-\frac{1}{\alpha}\right) x$ tends to 0 uniformly for $|x| \leq R$, there exists $N_{2}>0$ such that for $n>N_{2}$,

$$
\int_{|x| \leq R}\left|u_{0}\left(\frac{x}{\alpha}\right)-u_{0}\left(\frac{x}{\alpha_{n}}\right)\right| d x=\int_{|x| \leq R}\left|u_{0}\left(\frac{x}{\alpha}\right)-u_{0}\left(\frac{x}{\alpha}+h_{n}(x)\right)\right| d x<\frac{\varepsilon}{3} .
$$

Therefore we can conclude that $E_{3}^{\prime \prime} \rightarrow 0$ in $L^{1}$ :

$$
\begin{aligned}
\left\|E_{3}^{\prime \prime}\right\|_{1} & \leq \int_{|x|>R}\left(\left|u_{0}\left(\frac{x}{\alpha}\right)\right|+\left|u_{0}\left(\frac{x}{\alpha_{n}}\right)\right|\right) d x+\int_{|x| \leq R}\left|u_{0}\left(\frac{x}{\alpha}\right)-u_{0}\left(\frac{x}{\alpha_{n}}\right)\right| d x \\
& <\varepsilon \quad \text { for } \quad n>N_{1} \vee N_{2} .
\end{aligned}
$$

The proof is complete.

\section{REFERENCES}

1. L. Angiuli, D. Pallara, and F. Paronetto, Analytic semigroups generated in $L^{1}(\Omega)$ by second order elliptic operators via duality methods, Semigroup Forum 80 (2010), no. 2, 255-271.

2. D. Applebaum, Lévy processes and stochastic calculus, Cambridge Studies in Advanced Mathematics, Cambridge University Press, 2004.

3. V. Bally and L. Caramellino, On the distances between probability density functions, Electron. J. Probab. 19 (2014), no. 110, 1-33.

4. V. Bally and D. Talay, The law of the Euler scheme for stochastic differential equations (II): convergence rate of the density, Monte Carlo Methods Appl. 2 (1996), no. 2, 93-128.

5. H. S. Bhat and R. W. M. A. Madushani, Density tracking by quadrature for stochastic differential equations, Preprint, https://arxiv.org/abs/1610.09572v1 (2016).

6. J.-M. Bony, Principe du maximum dans les espaces de Sobolev, Comptes Rendus Acad. Sci. Paris, Série A 265 (1967), 333-336 (French).

7. N. Bouleau and F. Hirsch, Proprietes d'absolue continuite dans les espaces de dirichlet et application aux equations differentielles stochastiques, Séminaire de Probabilités XX 1984/85 1204 (1986), 131-161 (French).

8. Y. A. Butko, M. Grothaus, and O. G. Smolyanov, Feynman formulae and phase space Feynman path integrals for tau-quantization of some Lévy-Khintchine type Hamilton functions, J. Math. Phys. 57 (2016), no. 2, 023508, 22.

9. S. Cerrai, Analytic Semigroups and Degenerate Elliptic Operators with Unbounded Coefficients: A Probabilistic Approach, Journal of Differential Equations 166 (2000), no. 1, 151 174. 
10. W. Chai, A. Naess, and B. J. Leira, Stochastic Dynamic Analysis and Reliability of a Vessel Rolling in Random Beam Seas, Journal of Ship Research 59 (2015), no. 2, 113-131.

11. R. Cont and P. Tankov, Financial modelling with jump processes, Chapman and Hall/CRC Financial Mathematics Series, CRC Press, 2003.

12. M. G. Crandall, H. Ishii, and P.-L. Lions, User's guide to viscosity solutions of second order partial differential equations, Bull. Amer. Math. Soc. (N.S.) 27 (1992), no. 1, 1-67. MR 1118699

13. G. Da Prato and B. Goldys, Elliptic Operators on Rd with Unbounded Coefficients, Journal of Differential Equations 172 (2001), no. 2, 333 - 358.

14. K. J. Engel, R. Nagel, et al., One-parameter semigroups for linear evolution equations, Graduate Texts in Mathematics, Springer New York, 2006.

15. S. Fornaro and L. Lorenzi, Generation results for elliptic operators with unbounded diffusion coefficients in Lp- and Cb-spaces, Discrete and Continuous Dynamical Systems 18 (2007), no. $4,747-772$.

16. N. Fournier and J. Printems, Absolute continuity for some one-dimensional processes, Bernoulli 16 (2010), no. 2, 343-360.

17. M. G. Garroni and J. L. Menaldi, Second order elliptic integro-differential problems, Chapman \& Hall/CRC Research Notes in Mathematics Series, CRC Press, 2002.

18. D. Gilbarg and N. S. Trudinger, Elliptic partial differential equations of second order, Classics in Mathematics, Springer Berlin Heidelberg, 2015.

19. E. Gobet and C. Labart, Sharp estimates for the convergence of the density of the Euler scheme in small time, Electronic Communications in Probability 13 (2008), 352-363.

20. J. Guyon, Euler scheme and tempered distributions, Stoch. Process. their Appl. 116 (2006), no. 6, 877-904.

21. M. Hayashi, A. Kohatsu-Higa, and G. Yûki, Local Hölder Continuity Property of the Densities of Solutions of SDEs with Singular Coefficients, Journal of Theoretical Probability 26 (2012), no. 4, 1117-1134.

22. M. Hayashi and G. Kohatsu-Higa, A. Yûki, Hölder continuity property of the densities of SDEs with singular drift coefficients, Electron. J. Probab. 19 (2014), no. 76, 1-22.

23. E. R. Jakobsen and K. H. Karlsen, Continuous dependence estimates for viscosity solutions of fully nonlinear degenerate elliptic equations, Electron. J. Differ. Equations 2002 (2002), no. $39,1-10$.

24. P. E. Kloeden and E. Platen, Numerical Solution of Stochastic Differential Equations, Stochastic Modelling and Applied Probability, Springer Berlin Heidelberg, 1999.

25. V. Konakov and E. Mammen, Local limit theorems for transition densities of Markov chains converging to diffusions, Probability Theory and Related Fields 117 (2000), no. 4, 551-587.

26. V. Konakov and S. Menozzi, Weak Error for Stable Driven Stochastic Differential Equations: Expansion of the Densities, Journal of Theoretical Probability 24 (2010), no. 2, 454-478.

27. S. Kusuoka and D. Stroock, Applications of the Malliavin Calculus, Part II, J. Fac. Sci. Univ. Tokyo, Sect. IA, Math. 32 (1985), 1-76.

28. A. Lasota and M. C. Mackey, Chaos, fractals, and noise: Stochastic aspects of dynamics, Applied Mathematical Sciences, Springer New York, 1994.

29. V. Linetsky, The Path Integral Approach to Financial Modeling and Options Pricing, Computational Economics 11 (1998), no. 1-2, 129-63.

30. P.-L. Lions, A remark on Bony maximum principle, Proc. Amer. Math. Soc. 88 (1983), 503508.

31. C. Litterer and T. Lyons, High order recombination and an application to cubature on Wiener space, Ann. Appl. Probab. 22 (2012), no. 4, 1301-1327. MR 2985162

32. A. Lunardi, Analytic semigroups and optimal regularity in parabolic problems, Modern Birkhäuser Classics Series, Springer, 2012.

33. S. Menozzi and V. Lemaire, On Some non Asymptotic Bounds for the Euler Scheme, Electron. J. Probab. 15 (2010), no. 53, 1645-1681.

34. R. Metzler and J. Klafter, The restaurant at the end of the random walk: recent developments in the description of anomalous transport by fractional dynamics, J. Phys. A 37 (2004), no. 31, R161-R208.

35. E. Mo, Nonlinear Stochastic Dynamics and Chaos by Numerical Path Integration, Ph.D. thesis, Norwegian University of Science and Technology, 2008. 
36. A. Naess and J. M. Johnsen, Response statistics of nonlinear, compliant offshore structures by the path integral solution method, Probabilistic Engineering Mechanics 8 (1993), no. 2, 91-106.

37. B. Øksendal, Stochastic differential equations: An introduction with applications, Universitext, Springer Berlin Heidelberg, 2010.

38. P. Protter, Stochastic integration and differential equations, Stochastic Modelling and Applied Probability, Springer Berlin Heidelberg, 2005.

39. M. Rosa-Clot and S. Taddei, A path integral approach to derivative security pricing, II: Numerical methods, Cond-Mat/9901279, 1999.

40. C. Skaug and A. Naess, Fast and accurate pricing of discretely monitored barrier options by numerical path integration, Computational Economics 30 (2007), no. 2, 143-151.

41. J. S. Yu and Y. K. Lin, Numerical path integration of a non-homogeneous Markov process, International Journal of Non-Linear Mechanics 39 (2004), no. 9, 1493-1500, Themes in Nonlinear Stochastic Dynamics.

42. W. Zhang and J. Bao, Regularity of Very Weak Solutions for Nonhomogeneous Elliptic Equation, Communications in Contemporary Mathematics 15 (2013), no. 04, 1350012.

Norwegian University of Science and Technology, NO-7491, Trondheim, Norway

E-mail address: linghuac@math.ntnu.no

Norwegian University of Science and Technology, NO-7491, Trondheim, Norway

E-mail address: erj@math.ntnu.no

Norwegian University of Science and Technology, NO-7491, Trondheim, Norway

E-mail address: arvidn@math.ntnu.no 